\title{
Differential effects of sustained and transient effort triggered by reward - a combined EEG and pupillometry study
}

Mariam Kostandyan ${ }^{\star 1}$, Klaas Bombeke ${ }^{\star 1,2}$, Thomas Carsten ${ }^{1}$, Ruth M. Krebs ${ }^{1}$, Wim Notebaert ${ }^{1} \&$ C. Nico Boehler ${ }^{1}$

${ }^{1}$ Department of Experimental Psychology, Ghent University, Ghent, Belgium 2imec-mict-UGent, Ghent University, Ghent, Belgium

* these authors contributed equally to this work

Correspondence to:

C. Nico Boehler

Department of Experimental Psychology

Ghent University

Henri Dunantlaan 2

9000 Gent

Belgium

nico.boehler@ugent.be 


\section{Abstract}

In instrumental task contexts, incentive manipulations such as posting reward on successful performance usually trigger increased effort, which is signified by effort markers like increased pupil size. Yet, it is not fully clear under which circumstances incentives really promote performance, and which role effort plays therein. In the present study, we compared two schemes of associating reward with a Flanker task, while simultaneously acquiring electroencephalography (EEG) and pupillometry data in order to explore the contribution of effort-related processes. In Experiment 1, reward was administered in a block-based fashion, with series of targets in pure reward and noreward blocks. The results imply increased sustained effort in the reward blocks, as reflected in particular in sustained increased pupil size. Yet, this was not accompanied by a behavioral benefit, suggesting a failure of translating increased effort into a behavioral pay-off. In Experiment 2, we introduced trial-based cues in order to also promote transient preparatory effort application, which indeed led to a behavioral benefit. Again, we observed a sustained pupil-size increase, but also transient ones. Consistent with this, the EEG data of Experiment 2 indicated increased transient preparatory effort preceding target onset, as well as reward modulations of target processing that arose earlier than in Experiment 1. Jointly, our results indicate that incentive-triggered effort can operate on different time-scales, and that, at least for the current task, its transient (and largely preparatory) form is critical for achieving a behavioral benefit, which may relate to the temporal dynamics of the catecholaminergic systems.

Keywords: sustained pupil size, transient pupil size, reward, effort, EEG, CNV. 


\section{Introduction}

Reward is ever more recognized as a powerful modulator of cognition and behavior, both in terms of learning and in the sense of driving established behavior. As such, recent years have witnessed a steep increase in reports of specific task benefits of reward, ranging from cognitive control to attention to memory (for reviews, see Botvinick \& Braver, 2015; Krebs \& Woldorff, 2017). Although there is a multitude of potential mechanisms depending on how reward is administered (e.g., it can also be geared more towards perceptual/attentional learning mechanisms; Chelazzi, Perlato, Santandrea, \& Della Libera, 2013), one important way in which this happens is that reward prospect generally invigorates neural operations that are pertinent to the task at hand, which can even qualitatively change cognitive processes by promoting a more proactive control mode (Botvinick \& Braver, 2015; Braver, 2012; Notebaert \& Braem, 2015). Importantly, it is widely acknowledged that proactive control is effortful and limits the ability to perform other tasks simultaneously (Westbrook \& Braver, 2015, 2016).

Yet, there are numerous ways in which incentives can be associated with a task, and it is not clear under which incentive conditions effort is actually increased, and furthermore when such effort expenditure really leads to a behavioral benefit in rewardrelated tasks. Given that reward effects do not seem to arise ubiquitously (Bonner, Hastie, Sprinkle, \& Young, 2000), it seems possible that incentives do not always trigger appropriate effort adjustments (Camerer \& Hogarth, 1999; Jenkins Jr, Mitra, Gupta, \& Shaw, 1998); in addition, it could also be that sometimes effort is increased but in a way that is ultimately not successful at improving performance. Based on the fact that effort is inherently aversive, and is usually discounted from the reward that can be obtained in a given task (Kool, McGuire, Rosen, \& Botvinick, 2010; Shenhav, et al., 2017; Verguts, Vassena, \& Silvetti, 2015; but see also Inzlicht, Shenhav, \& Olivola, in press), a failure of translating effort into a benefit (in the broad sense) would seem particularly problematic on a theoretical level. Rather, one would expect that effort would usually need to yield some benefit in order to compensate for the general aversiveness.

Importantly, differences in how reward is associated with a task might have consequences for how a reward benefit is brought about (Krebs, Hopf, \& Boehler, 2016). 
Most studies employ cues predicting the potential of reward in the upcoming trial (e.g., Padmala \& Pessoa, 2011; van den Berg, Krebs, Lorist, \& Woldorff, 2014), whereas other studies directly associate specific task features with reward (e.g., specific rewarded ink colors in Stroop tasks; Krebs, Boehler, Egner, \& Woldorff, 2011). Yet others have used block-wise reward manipulations (e.g., Kouneiher, Charron, \& Koechlin, 2009; Locke \& Braver, 2008; Massar, Lim, Sasmita, \& Chee, 2016; Paschke, et al., 2015). In the present work, we compared a block-based incentive condition with one that used cues on every trial. To this end, we combined pupillometry and EEG measures to gain insights into the dynamics of reward- and effort-related processes in these task contexts.

Pupil size has long been considered as a correlate of mental effort, increasing in various contexts of enhanced task difficulty that are largely devoid of any other factors that are relevant for pupil size, such as affective aspects (Beatty, 1982b; Kahneman \& Beatty, 1966; van der Wel \& van Steenbergen, 2018). In this context, it is important to note that pupil-size modulations arise on different temporal scales, displaying both sustained/tonic and transient/phasic changes, both of which have been related to effort (Beatty, 1982a; Chiew \& Braver, 2013). Pupil size is furthermore linked through a lot of evidence to activity in the norepinephrinergic (NE) system, and seems generally related to arousal and the autonomous nervous system (for a review, see Eckstein, GuerraCarrillo, Singley, \& Bunge, 2017). However, direct inferences from physiological parameters such as pupil size to a psychological construct such as effort are difficult (Richter \& Slade, 2017) and, depending on the task and a participant's reaction to it, other arousal-related factors could play a role, such as emotional aspects (Bradley, Miccoli, Escrig, \& Lang, 2008).

Concerning EEG activity, we were particularly interested in processes related to target selection, reported before as a reward-related ERP difference in the form of relative positivities over fronto-parietal areas (Krebs, Boehler, Appelbaum, \& Woldorff, 2013; van den Berg, et al., 2014). Additionally, in the cuing context, we were interested in processes that are triggered by the cue and relate to preparatory effort, in particular the contingent negative variation (CNV) event-related potential (ERP) component (van den Berg, et al., 2014). Additionally, we wanted to explore the role of early sensory processes during task stimulus processing. In order to disentangle the processing of the distracters and that of 
the target, we adopted the paradigm of Appelbaum and colleagues, in which there was a $200 \mathrm{~ms}$ stimulus onset asynchrony (SOA) between the task-irrelevant and task-relevant information (Appelbaum, Boehler, Won, Davis, \& Woldorff, 2012). Given that hence irrelevant (and detrimental information in case of incongruent trials) was consistently presented first, we expected that reward would lead to diminished distracter processing as reflected by the attentional N1 ERP component (see also Bombeke, Langford, Notebaert, \& Boehler, 2017).

In order, to investigate the effects of reward on sustained and transient effort, we conducted two separate experiments. Initially, we ran an EEG-pupillometry experiment investigating pure block-based effects of reward (Experiment 1). Based on the results of this experiment, and in particular the lack of a behavioral reward effect therein, this was followed up by an experiment that employed cues to communicate reward availability (Experiment 2). Experiment 2 featured reward blocks with trial-based cues predicting whether successful performance on a given trial could garner a monetary bonus or not, as is typical in this set-up (e.g., Padmala \& Pessoa, 2011), and a pure no-reward block with cues consistently predicting that no extra bonus could be earned. Therefore, Experiment 2 allowed to investigate both block effects (as in Experiment 1), and eventrelated ones (reward-related vs. no-reward trials in the reward blocks), as has been successfully demonstrated before in this general context (e.g., Chiew \& Braver, 2013; Jimura, Locke, \& Braver, 2010).

In both experiments, we expected behavioral reward benefits (as already previewed, this was ultimately not found in Experiment 1), and assumed that they would go together with markers of effort, in particular pupil size, both concerning sustained and transient modulations (Chiew \& Braver, 2013). Moreover, we expected modulations of attentional processing of the distracter- and target-stimulus. Specifically, we hypothesized to find that less attention is devoted to the distracter-stimuli under reward, whereas we expected enhanced processing of the full stimulus (distracters plus target). Specific to Experiment 2, we predicted correlates of preparatory effort to be increased by reward information, in particular the cue-related CNV, as well as indications of preparatory effort in the pupil-size data, such as cue-locked increases, likely extending into target processing. 


\section{Methods}

\section{EXPERIMENT 1}

Participants. Twenty-three participants (seven men, age $M=22.8$, all right-handed) with no history of psychiatric or neurological disorders and with normal or corrected-to-normal vision took part in Experiment 1. They received a compensation of $€ 25$ for a session that lasted approximately two hours, plus a variable performance-based monetary bonus of up to $€ 5$. One participant was excluded from the analysis due to noisy EEG data. Prior to the beginning of the experiment all participants gave a written informed consent. The study was approved by the ethical committee of the Faculty of Psychology and Educational Sciences of Ghent University.

Stimuli and design. We used a variant of the Flanker task with arrows pointing in four different directions (left up, left down, right up, right down). White arrows were presented on a gray background with a small fixation dot at the center of the screen. We presented the irrelevant distracter arrows 200 ms before the relevant target arrows (Appelbaum, et al., 2012), which allowed for a 200-ms analysis window of the EEG with pure distracter processing. Two independent variables were manipulated: 1) congruency - trials could be either congruent, e.g., distracter stimuli and target-stimulus both pointing upwards left, or incongruent, e.g., distracter stimuli pointing downwards left and target-stimulus pointing upwards right (we distributed twelve possible pairings of distracter-target evenly); and 2) reward - each block was either reward-related or a pure no-reward block. Participant responded by pressing one of the QWERTY keyboard's button: "A" (arrow directed to the up left side), "Z" (down left), "K" (up right), and "M" (down right).

All trials started with the onset of distracters for $200 \mathrm{~ms}$ (see Figure 1A). Next, the target arrow appeared for $200 \mathrm{~ms}$ in the middle of the screen, between the distracters. Participants were instructed to respond to the direction of the target arrow as fast and accurately as possible. Because of the consistent timing, participants knew that the irrelevant arrows would always be presented first. Following target offset, an inter-trial interval with just a fixation dot was presented for a variable duration. This duration was the sum of the time it took for participants to provide their response with a maximum of 
$1000 \mathrm{~ms}$ concerning the duration of this stimulus event (note that this is independent of the response deadline, see below) plus an additional randomly-jittered duration of 900 $1200 \mathrm{~ms}$ ( $M=1050 \mathrm{~ms}$ ). Importantly, participants were informed that they would get additional monetary rewards based on a point scheme (+10 points) for each trial when responding fast and accurately in reward-related blocks. In the reward-related blocks, cumulative reward feedback in the form of the sum of earned points was presented every 20 trials. In order to keep this challenging and comparable across participants, an adaptive procedure constantly adjusted the time-out of the response-time window. This procedure adjusted the response time-out by $20 \mathrm{~ms}$ every time the cumulative reward rate over the course of the experiment would cross $65 \%$ (i.e., extend it by $20 \mathrm{~ms}$ when it fell below $65 \%$, and decrease it by $20 \mathrm{~ms}$ when it exceeded $65 \%$ ). Ultimately, this procedure was successful and yielded very similar reward rates for all participants. Reward rates mostly only differed to a minor degree as a function of accuracy (since incorrect trials were not rewarded). At the end of the experiment the total number of earned points was converted to a monetary bonus.

Participants completed four fully randomized blocks of 128 trials each (64 congruent and 64 incongruent trials) half of which could yield reward, while the other half could not $(R B=$ reward-related block, $N R B=$ no-reward block). The RB consisted only of reward-related trials (RBrew) and the NRB contained only no-reward trials (NRB). The presentation order of blocks was counterbalanced across participants (RB-NRB-RB-NRB vs. NRB-RB-NRB-RB) in order to minimize the influence of training effects or spill-over effects (see Figure 1B). 
A

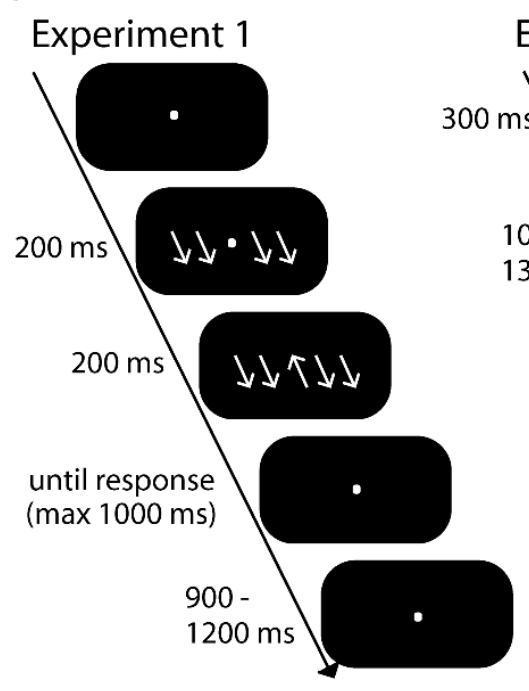

Reward-related block, reward (RBrew) No-reward block (NRB)
B

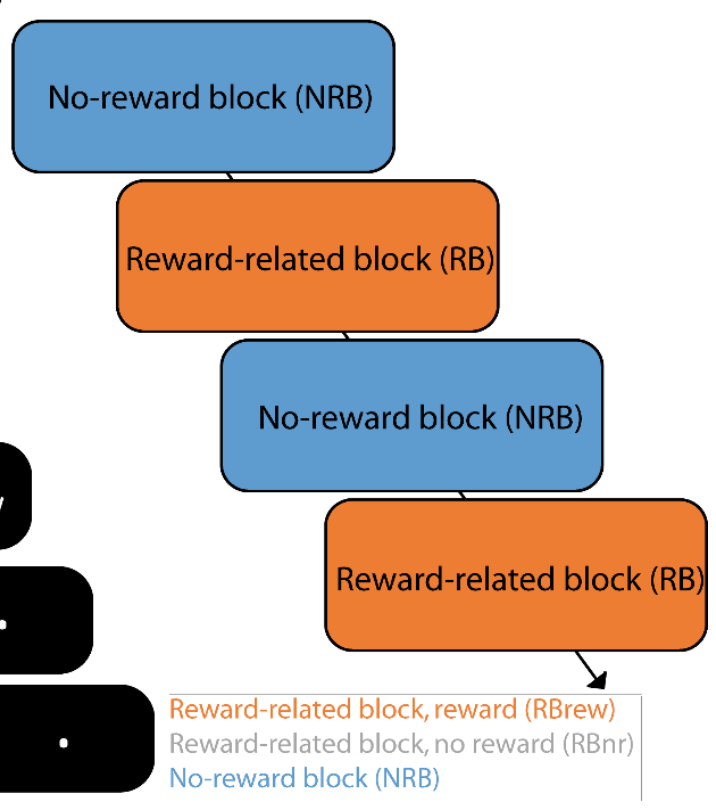

o-reward block (NRB)
Experiment 2

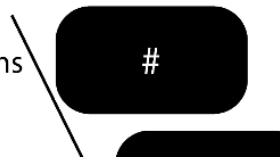

\section{.}

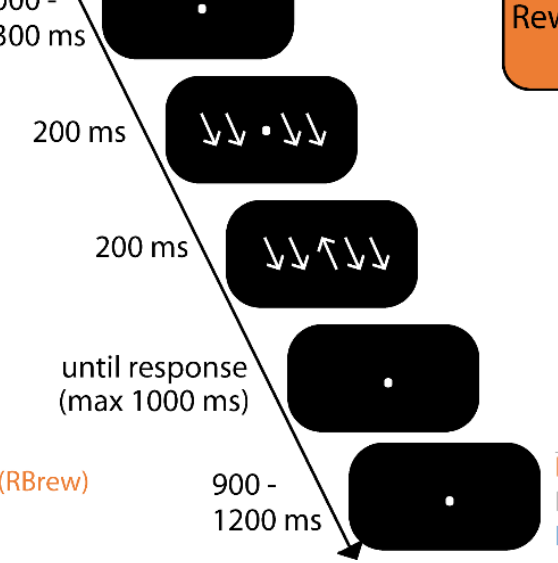

Figure 1. Paradigm. (A) On each trial, participants were instructed to manually respond to the direction of the target arrow as fast and accurately as possible. Irrelevant distracter arrows preceded the relevant target arrow by $200 \mathrm{~ms}$. At the bottom, the different conditions of each experiment are mentioned. (B) In both experiments trials were presented in alternating blocks (either RB or NRB) counterbalanced across participants.

Behavioral analysis. For RT analyses, all incorrect and missed responses were excluded, and only correct responses within a time window 200-1400 ms were included in the analysis (irrespective of the adaptive response time-out procedure employed to adjust reward rates). RT and error rates were analyzed with repeated-measures ANOVAs (rANOVAs), with factors reward (RB vs. NRB) and congruency (congruent vs. incongruent trial). For response-time measures, the RT is reported time-locked to the onset of the target arrow. Effect sizes are reported as partial eta squared.

Pupil measurements and preprocessing. We used a $250 \mathrm{~Hz}$ SMl eye tracker (RED250 mobile system; SensoMotoric Instruments, Teltow, Germany) to continuously measure pupil size of both eyes during the experiment. A camera with infrared optics was attached to a standard 17-inch computer screen and a chinrest was used to minimize head movements and to maintain a fixed distance of $65 \mathrm{~cm}$ from the screen. Each block of the experiment started with a calibration procedure in which participants had to follow a moving red dot with their eyes to nine locations on a grey background, the success of 
which was validated before continuing. The EEG chamber was dimly lit constantly throughout the experiment. Short stretches of missing data points due to blinks or recording errors were corrected by means of a linear interpolation procedure. Pupil size was initially recorded at a sampling rate of $250 \mathrm{~Hz}$, but then up-sampled to $500 \mathrm{~Hz}$ to match the sampling rate of the EEG (see below). Trigger codes in both the pupil and EEG data were synchronized with the EYE-EEG Matlab toolbox (Dimigen, Sommer, Hohlfeld, Jacobs, \& Kliegl, 2011). As a first preprocessing step, a raw data inspection was performed, excluding stretches with clear artifacts as well as portions of the data with significant data loss. This procedure mostly excluded flat stretches resulting from the linear interpolation of periods with a lot of blinks. The raw data inspection was done in a fashion that was blind towards the specific task conditions, and generally led to only very minor data exclusion (less than $3 \%$ of the continuous raw data). The data was then epoched from -200 ms to $2000 \mathrm{~ms}$, time-locked to the onset of the distracter-stimulus and averaged afterwards per participant according to the main experimental conditions (keeping only the correct trials). In an analysis targeted at sustained effects, absolute pupil size was used, without applying baseline correction. For this, we used a 400-ms time-window around the distracter-stimulus onset, starting $200 \mathrm{~ms}$ before distracter onset and ending with target-stimulus onset, as an estimate of sustained pupil size that was still unaffected by the subsequent light reflex and the condition of the trial. In this analysis, we additionally used block-order (RB-NRB-RB-NRB or NRB-RB-NRB-RB) as a co-variate to account for a block-independent decrease in pupil size over time (see results). To look at transient pupil-size modulations above and beyond simultaneous sustained effects, we baseline-corrected the data with regard to the pre-distracter-stimulus baseline. This data was analyzed in a time-resolved fashion by using adjacent 100-ms time-windows from distracter-stimulus onset until $2000 \mathrm{~ms}$ thereafter, running a rANOVA in each window with factors reward (RB vs. NRB) and congruency (congruent vs. incongruent). Statistical significance is reported using both uncorrected $p$-values as well as by using falsediscovery rate (FDR) correction (Benjamini \& Hochberg, 1995). Ultimately, after confirming that the data never differed in a significant way across the two eyes, data was usually collapsed across both eyes, with few exceptions where the data from one eye had 
clearly lower data quality (more data loss), in which case information was used only from the other eye (three participants in Experiment 1).

EEG acquisition, preprocessing and analysis. EEG data were collected using a Brain Products actiCHamp 64-channel system (Brain Products, Gilching, Germany) with 64 active scalp electrodes positioned according to the standard international 10-20 system, two of which were attached to the left and right mastoids. Signals were recorded in the reference-free mode with a sampling rate of $500 \mathrm{~Hz}$. The EEG data was analyzed using Brain Vision Analyzer 2 (Brainproducts, Gilching, Germany). During preprocessing, data was re-referenced offline to the average of the mastoids, followed by a raw-data inspection to exclude stretches of clearly identifiable bad data. Next, a band-pass filter of $0.02-40 \mathrm{~Hz}$ was applied. After that, we removed blinks from the data using independent component analysis (ICA). The data were then epoched from -200 ms to $1000 \mathrm{~ms}$, timelocked to the onset of the distracter-stimulus, and a semi-automatic artifact rejection was performed in a fashion that was completely blind to the different experimental conditions. Afterwards, the data was again epoched, now with regard to the actual conditions, and again limited to correct trials. Afterwards, the data was baseline-corrected using the 200 ms period preceding distracter-stimulus onset, and the data was averaged per participant.

The EEG data was analyzed with regard to two main components. First, we investigated possible $\mathrm{N} 1$ modulations related to distracter-stimulus processing. For this, we used the average across conditions to determine the most representative channels and time-range leading us to average P5, P3, PO3, PO7, P6, P4, PO4, PO8 between 130 and $200 \mathrm{~ms}$. In addition, we looked at a fronto-central reward modulation; the ROI was based on earlier work (van den Berg, et al., 2014) and contained channels FC1, FCz, $\mathrm{FC} 2, \mathrm{C} 1, \mathrm{Cz}, \mathrm{C} 2$, on the average of which we performed tests in adjacent 50-ms windows between 300 and $700 \mathrm{~ms}$ to explore the temporal evolution, similar to the analysis of the transient pupil responses, but limiting ourselves to only the factor reward. Once again, FDR-corrected $p$-values are reported in addition to the uncorrected ones.

Finally, in an exploratory analysis driven by the results of the planned main analyses, we tried to pinpoint block-level reward-related differences in EEG activity that would potentially mirror the sustained pupil-size differences that we observed. Based on an earlier report that identified such a relationship (Hong, Walz, \& Sajda, 2014), we 
focused on alpha power as an electrophysiological correlate of attentional task disengagement prior to a trial (Babiloni, et al., 2006; van Dijk, Schoffelen, Oostenveld, \& Jensen, 2008). We derived alpha power by using the complex-demodulation function of Brain Vision Analyzer 2 (extracting power values averaged between 8 and $12 \mathrm{~Hz}$ ), which we then analyzed in a fashion that was analogous to the analysis of sustained pupil size. Specifically, we focused on 500-ms time windows preceding distracter-stimulus presentation as an estimate for block-level effects, which we did not baseline-correct, in order to identify sustained effects. Based on the average of all conditions, we averaged alpha power across $\mathrm{POz}$ and $\mathrm{Pz}$ where raw alpha power was maximal in both experiments (i.e., irrespective of any condition-wise differences; see inserts in Figure 7), and tested for condition-wise differences. In addition, given that alpha power tends to increase with time-on-task as a function of decreases in vigilance (Cajochen, Brunner, Krauchi, Graw, \& Wirz-Justice, 1995), we included block order as a covariate into our analyses, equivalent to our approach for sustained pupil size.

\section{EXPERIMENT 2}

The paradigm and analysis of Experiment 2 paralleled those of Experiment 1 as best as possible. We therefore mostly limit ourselves to aspects that differed across the experiments.

Participants. Twenty-four participants (seven men, age $M=23.5$, all right-handed) with no history of psychiatric or neurological disorders and with normal or corrected-to-normal vision that had not participated in Experiment 1 took part in the Experiment 2. They received a monetary compensation of $€ 25$ for a two-hour session, with again up to $5 €$ performance-contingent bonus. The first two participants had to be excluded due to the technical problems with excessive eye-tracking data loss and with sending port codes, respectively.

Stimuli. We used the same Flanker stimuli and task as in the Experiment 1. In addition, three different symbols (“@”, "\#”, and "§”) were used to communicate the possibility to win reward on each trial. These cues were chosen to not be inherently associated with the concept of money. In the counterbalancing procedure across participants each 
symbol was used as a reward-related cue in the $\mathrm{RB}$, a no-reward cue in the $\mathrm{RB}$, and a no-reward cue in the NRB.

Procedure and design. Each trial started with one of the cues that appeared for $300 \mathrm{~ms}$ predicting the possibility to earn an extra reward in case of a correct response (see Figure 1A). The cue was followed by a blank interval randomly varying between 1000 and 1300 ms during which only the fixation square remained on the screen. Next, the distracters appeared for $200 \mathrm{~ms}$ followed by the target-stimulus appearing in the center of the screen for $200 \mathrm{~ms}$. After, displaying a fixation dot until the participant's response (or a maximum of $1000 \mathrm{~ms}$ ), the same display was kept for another randomly-varied 900 to $1200 \mathrm{~ms}$.

The experiment consisted of two reward-related blocks of 128 trials each (64 congruent and 64 incongruent trials), and two no-reward blocks with 64 trials each. In both RB there were 64 reward-related trials (RBrew) and 64 no-reward trials (RBnr). NRB blocks consisted of 64 no-reward trials (NRB), hence matching the number of RBrew and RBnr trials, respectively. Because we wanted to keep the overall monetary bonus the same across the two experiments, and there were only half as many reward-related trials in Experiment 2, each reward-related trial was worth more money in Experiment 2 than in Experiment 1; however, the use of an independent sample of participants, the cumulative nature of the reward feedback, and the indirect link between the point scheme and the payout make it unlikely that this played a major role in bringing about differences between the two experiments. As in the Experiment 1, the order of blocks was counterbalanced across participants (RB-NRB-RB-NRB vs. NRB-RB-NRB-RB).

Behavioral data acquisition and analysis. The same basic analysis was performed as in Experiment 1. Given the complicated set-up of Experiment 2, however, featuring both across-block and within-block comparisons, we used three separate rANOVAs, looking at: 1) across-block reward effects (NRB vs. RBrew trials as in Experiment 1), 2) at withinblock reward effects (RBrew vs. RBnr trials) and 3) the across-block context effects for the different types of no-reward trials (NRB vs. RBnr trials). This general approach has been successfully used in related earlier work (Chiew \& Braver, 2013; Jimura, et al., 2010). 
Pupil measurements and preprocessing. We used the same eye-tracking set-up and preprocessing as in the Experiment 1. The pupil data was analyzed in the same way as for Experiment 1, with the addition of a cue time-range. There, we extracted epochs with the same length (-200 to $2000 \mathrm{~ms}$ ), but during the analysis excluded data beyond 1600 ms post-distracter-stimulus to avoid any effect of the subsequent target processing. Data quality was comparable across both eyes for all participants, leading us to collapse the data across eyes for all participants.

EEG acquisition, preprocessing and analysis. Data was recorded from the same 64 active scalp electrodes as in Experiment 1. In contrast to the reference-free recording in Experiment 1, the data was now referenced online against a reference channel. For different participants, that was either $\mathrm{Fz}$ or $\mathrm{FCz}$, which were later recovered back into the data during re-referencing to the average mastoids. Note that due to the linear nature of the re-referencing procedure, the online reference is rather arbitrary, and the resulting datasets all contained exactly the same channels. The basic analysis replicated that of Experiment 1, with the addition of the cue time-window. For this, a time-window between -200 and $1500 \mathrm{~ms}$ around the onset of the cue-stimulus was used. Given that it is unclear at which stage processing goes awry in incorrect trials, with only a subset of such failures likely directly relating to absent task preparation, for the cue analysis (in contrast to the target analysis), all trials were kept irrespective of whether ultimately the target was responded to correctly or not.

The EEG data for the target time-range was analyzed analogously to Experiment 1. In addition, we looked at two cue-related components, the P3 and the CNV. The ROls were determined by the average across all conditions (P3, POz, P4 for the P3, and FCz and $\mathrm{Cz}$ for the CNV). Given that both effects of interest are most visible as differences between conditions, we used time-windows of earlier work that have looked at such differences. Specifically, we used a time-window between 300 and $500 \mathrm{~ms}$ post-cue for the P3 (Goldstein, et al., 2006; Schevernels, Krebs, Santens, Woldorff, \& Boehler, 2014) and a time-window for the CNV that started 700 ms post-cue (Schevernels, et al., 2014; van den Berg, et al., 2014) and lasted until the end of the cue-target interval at $1500 \mathrm{~ms}$. 
Alpha power was analyzed in the same way as in Experiment 1 preceding distracterstimulus presentation, but also in an equivalent way preceding cue presentation.

Across-experiment comparisons. In addition to the within-experiment comparisons, we also sought to directly compare results across experiments where possible (Nieuwenhuis, Forstmann, \& Wagenmakers, 2011). Depending on the exact analysis, this was done by including experiment as a between-subjects factor in a rANOVA, or by running a twosamples t-test. Given power limitations, we combined the data of Experiment 2 across corresponding conditions wherever there was no clear difference.

\section{Results}

Whereas we will present the results of the two experiments in sequence, they are combined in the same figure where possible, in order to facilitate direct comparisons.

\section{EXPERIMENT 1}

\section{Behavior}

The left panels of Figure 2 display the behavioral data of Experiment 1. A two-way rANOVA with factors congruency (congruent vs. incongruent) and reward (NRB vs. RBrew) on the average response times showed a highly significant main effect of congruency $\left(F(1,21)=210.4 ; p<0.001 ; \eta_{p}^{2}=0.91\right)$, but no significant main effect of reward $(p>0.8)$. The interaction between congruency and reward context was also not significant $(p>0.6)$. Accuracy analysis showed a result pattern that was similarly dominated by congruency but unaffected by reward. The main effect of congruency was again highly significant $\left(F(1,21)=46.96 ; p<0.001 ; \eta_{p}^{2}=0.69\right)$, whereas the effect of reward $(p>0.75)$ and the interaction between congruency and reward were not $(p>0.4)$.

\section{Pupil size}

Sustained pupillary response. Given the block design of reward-related and no-reward blocks, we first interrogated the data for sustained block-level effects using the data before baseline correction, and focusing on a 400-ms window around distracter-stimulus onset as an estimate of sustained activity independent of the actual events. Since pupil 
size is very sensitive for time-on-task and tends to decrease globally over the course of an experiment (e.g., Massar, et al., 2016), we checked whether there was a block-typeindependent decrease by averaging all events in the first and second half of the experiment. This average hence represents the average of an RB and an NRB early and late in the experiment, and indeed showed a consistent effect in the expected direction with larger pupils in the first than the second half of the experiment (5.62 vs. $5.54 \mathrm{~mm}$ respectively; $p=0.006$ ). We therefore included block order (RB-NRB-RB-NRB vs. NRBRB-NRB-RB) as a covariate into analyses of sustained pupil size (note that transient evoked responses showed no such effect across the halves of the experiment).

Using the 400-ms time-window around distracter-stimulus onset to probe for sustained differences in pupil size (see Figure $3 \mathrm{~A}$, left panel), pupil-size was clearly different across the two block types (RB: 5.65 vs. NRB: $5.49 \mathrm{~mm}$ ), and a rANOVA with factors reward and congruency, as well as the co-variate of block order, yielded a highly significant main effect of reward $\left(F(1,20)=10.96 ; p=.003 ; \eta_{p}^{2}=0.35\right)$. As expected, congruency did not play a role at this point, showing neither a main effect nor an interaction with reward (both $p>0.25$ ).

Transient pupillary response. For analyzing transient pupillary effects above and beyond sustained block differences, the data was baseline-corrected and interrogated for differences in adjacent windows of 100 ms between distracter-stimulus onset and 2000 $m s$ thereafter, in order to also get insights into the temporal dynamics (see Figure 3B, left panel). In those 100-ms windows, we performed a two-way rANOVA with factors congruency and reward. The result pattern indicated a consistent absence for the main effect of reward (uncorrected $p$-values between 0.07 and 0.52 ; all FDR-corrected $p>0.4$ ) and for the interaction of reward and congruency (all uncorrected $p>0.15$; all FDRcorrected $p>0.9$; interaction not plotted in Figure $3 \mathrm{~B}$ ). In contrast, there were strong effects of congruency throughout most of the investigated time-window (uncorrected $p$ values between 0.00001 and 0.08 ; FDR-corrected $p$-values between 0.0002 and 0.08 ). Interestingly, that included an early effect with four consecutive tests between 0 and 400 ms being significant (uncorrected $p$-values between 0.008 and 0.034 ; FDR-corrected $p$ - 
values between 0.016 and 0.043$)^{1}$, and a later time-window between 800 and $2000 \mathrm{~ms}$ (uncorrected $p$-values between 0.00001 and 0.03; FDR-corrected $p$-values between 0.0002 and 0.041 ). The timing of the very pronounced late effect is consistent with the latency of earlier reports of incongruency-related pupil-size changes (Laeng, Orbo, Holmlund, \& Miozzo, 2011).

\section{EEG}

Distracter-related effects - N1

In a first analysis, we looked at attentional effects during the first $200 \mathrm{~ms}$ that are exclusively related to distracter processing, with a focus on the $\mathrm{N} 1$ component (see Figure $5 A)$. For this we analyzed the data from the channels and the time-range representative of the average N1. This revealed no clear significant effect for reward $(t(21)=1.83 ; p=$ $0.08)$, suggesting that early attentional processing was not clearly modulated by reward. Note that congruency is not a factor at this point, since the target arrow is present only afterwards.

\section{Post-target fronto-central reward difference}

We furthermore investigated reward-related differences in a fronto-central $\mathrm{ROI}$ comprising $\mathrm{FC} 1, \mathrm{FCz}, \mathrm{FC} 2, \mathrm{C} 1, \mathrm{Cz}, \mathrm{C} 2$. In this $\mathrm{ROI}$, comparing RBrew vs. NRB trials in adjacent 50-ms windows between 300 and $700 \mathrm{~ms}$ with paired-samples t-tests (see Figure 6, top panel) yielded significant differences between 500 and $700 \mathrm{~ms}$ (uncorrected $p$-values between .001 and .011; FDR-corrected $p$-values between 0.01 and 0.02 ).

\section{Baseline alpha power}

Finally, we investigated whether reward-related block-level differences preceding distracter-stimulus processing manifested in the EEG data, akin to the sustained effects of pupil size. For this, we investigated alpha power in a 500-ms time window preceding

\footnotetext{
${ }^{1}$ This early modulation by conflict is surprising; given that it starts before congruency is even established on a given trial (plus the sluggishness of the pupil response), this (small) modulation must reflect some other process. Specifically, it might relate to slight differential overlap from the previous trial (e.g., also in a fully random sequence, it could coincidentally happen that e.g., incongruent trials are slightly more frequently preceded by incongruent than congruent trials), and/or to slight imbalances in trial numbers (due to the exclusion of incorrect trials, there are slightly more congruent than incongruent trials in the analysis).
} 
distracter onset in both blocks, without baseline-correcting it (Figure 7A). Indeed, we observed lower amplitudes of alpha power preceding distracter-stimulus onset in the RB vs. NRB blocks $\left(F(1,20)=11.53 ; p=0.003 ; \eta_{p}^{2}=0.37\right)$, likely indicating a higher state of attention/vigilance globally in the RB blocks.

\section{EXPERIMENT 2}

\section{Behavior}

In order to capture all possible reward effects in this paradigm, we used three separate two-way rANOVAs featuring factors of congruency and reward, the latter of which taking three different forms. Specifically, separate analyses aimed at looking at the across-block reward effects (NRB vs. RBrew), within-block reward effects (RBrew vs. RBnr), and an across-block context effects for the different types of no-reward trials (NRB vs. RBnr; see e.g., Chiew \& Braver, 2013; Jimura, et al., 2010).

Response times. Probing for across-block reward effects corresponding to Experiment 1 (NRB vs. RBrew), we again observed a clear main effect of congruency $(F(1,21)=207.26$; $\left.p<0.001 ; \eta_{p}^{2}=0.91\right)$ whereas the main effect reward did not reach significance $(F(1,21)$ $\left.=3.17 ; p=0.089 ; \eta_{p}^{2}=0.13\right)$. Yet, given the two-tailed nature of the test is not fully consistent with our directed hypothesis, we still consider this as supporting the presence of a reward effect. Finally, the two factors did not interact significantly $(p>0.85)$. Turning to within-block reward effects (RBrew vs. RBnr), clear main effects were found for congruency $\left(F(1,21)=185.7 ; p<0.001 ; \eta_{p}^{2}=0.89\right)$ and also for reward $(F(1,21)=17.2$; $\left.p<0.001 ; \eta_{p}^{2}=0.45\right)$ were found, but no interaction $(p>0.9)$. Looking for across-block context effects (NRB vs. RBnr) in a corresponding rANOVA revealed a comparable main effect of congruency $\left(F(1,21)=185.9 ; p<0.001 ; \eta_{p}^{2}=0.9\right)$, but no main effect of reward or interaction (both $p>0.9$ ), indicating that the RBnr trials did not profit behaviorally from the general reward context when compared to NRB trials.

Accuracy data. All three rANOVAs yielded significant main effects of congruency (all $p<$ 0.05 ), but no main effects of reward or interaction of the factors (all $p>0.4$ ). In sum, the behavioral data indicate typical effects of congruency across blocks and conditions, with faster and more accurate responses for congruent than incongruent trials. Reward, in 
contrast, had a specific effect on the response speed in RBrew trials only. Finally, accuracy was not affected significantly by reward, suggesting that the effects on response speed were not strongly driven by differential speed-accuracy trade-off.

A

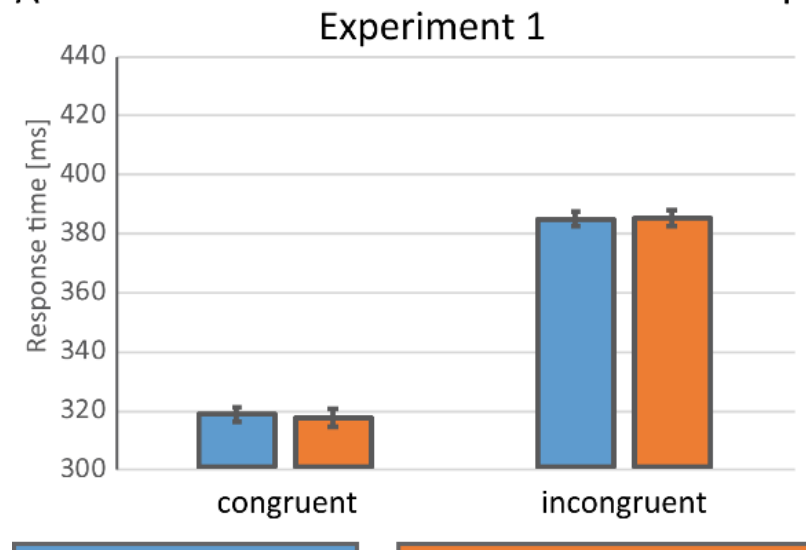

Response time

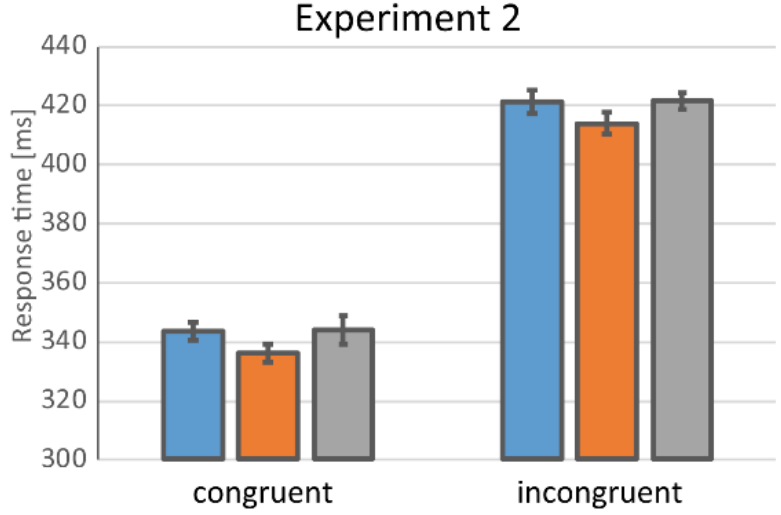

No-Reward block (NRB)

Reward-related block, reward (RBrew)

Reward-related block, no reward (RBnr)

B

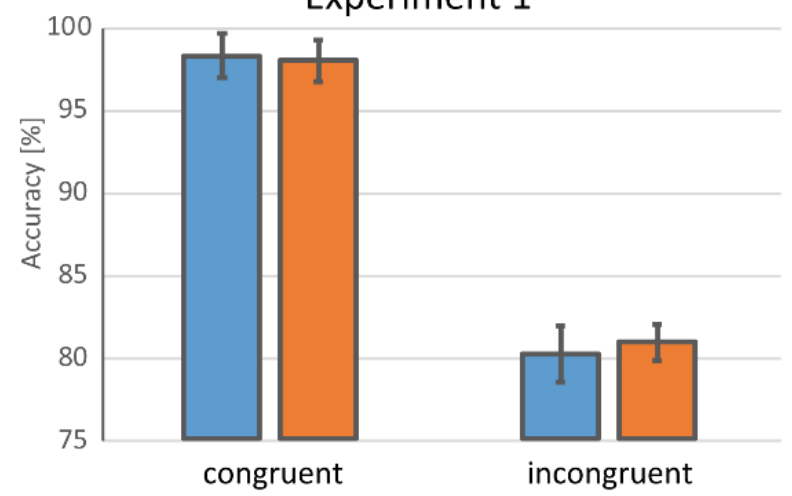

Accuracy

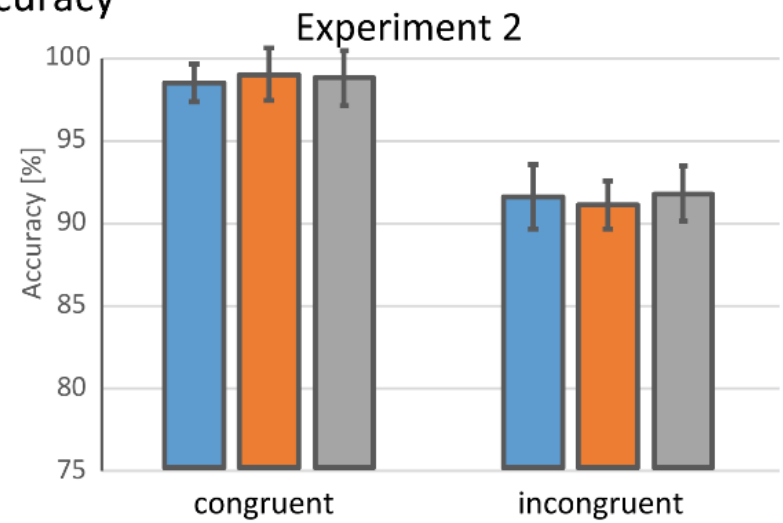

Figure 2. Behavioral results. A) Response-time results for Experiment 1 (left) and Experiment 2 (right). Both experiments showed clear effects of congruency, but only Experiment 2 displayed a reward benefit. B) Accuracy results. Both experiments showed clear effects of congruency, but neither displayed effects of reward. Error bars represent the within-subject standard error of the mean (Morey, 2008).

\section{Pupil size}

Sustained effects. We again first interrogated the data for sustained block-level effects using the raw data before baseline correction. As a first step, we checked whether there was again a block-type-independent pupil-size decrease over time, by averaging all cue and target-stimulus events in the first and second half of the experiment. This average indeed showed a consistent effect with larger pupils in the first than the second half of the 
experiment (5.43 vs. $5.31 \mathrm{~mm}$ accordingly), which approached statistical significance ( $p$ $=0.051$ ). Therefore, and in order to stay consistent with the analysis in Experiment 1, we decided to again include block order (RB-NRB-RB-NRB vs. NRB-RB-NRB-RB) as a covariate into analyses of sustained pupil size (note that transient evoked responses again showed no such effect). Using a simple one-way rANOVA additionally including this covariate for the cue time-window (see Figure $3 \mathrm{~A}$, right panel), we found strong differences between both trial types from the RB and the NRB (RBrew vs. NRB: $F(1,20)$ $=26.1 ; p<0.00001 ; \eta_{p}^{2}=0.57 ;$ RBnr vs. NRB: $\left.F(1,20)=24.68 ; p<0.00001 ; \eta_{p}^{2}=0.55\right)$, whereas the difference between RBrew and RBnr was not significant $(p>0.7)$. Similar to this, there was a strong effect of block type on sustained activity for the target data (see Figure $3 \mathrm{~A}$, middle panel), again reflected in strong differences of the two RB trial types against the data from the NRB (RBrew vs. NRB: $F(1,20)=20.9 ; p=0.0002 ; \eta_{p}^{2}=0.51$; RBnr vs. NRB: $\left.F(1,20)=20.56 ; p=0.0002 ; \eta_{p}^{2}=0.51\right)$, with no significant difference between RBrew and RBnr $(p>0.9)$. This analysis furthermore included the factor congruency, which however showed no significant main effects or interactions (all $p>$ $0.05)$, with the exception of a slight interaction between reward and congruency in the RBrew vs. RBnr comparison $\left(F(1,20)=4.5 ; p=0.047 ; \eta_{p}^{2}=0.18\right)$. Given that congruency is not even a factor at this point (the target arrow gets presented after the end of the quantified interval), we consider this either spurious or reflecting a slight imbalance in previous trial type and/or in trial numbers due to the exclusion of incorrect trials (see also footnote 1). In summary, there were very consistent block effects that mostly did not seem to interact with the actual conditions in those blocks (i.e., it did not matter whether trials in the reward block were actual reward trials or not, as well whether they were congruent or not), as one would expect given that this information will only be reflected in the pupil with a delay. 

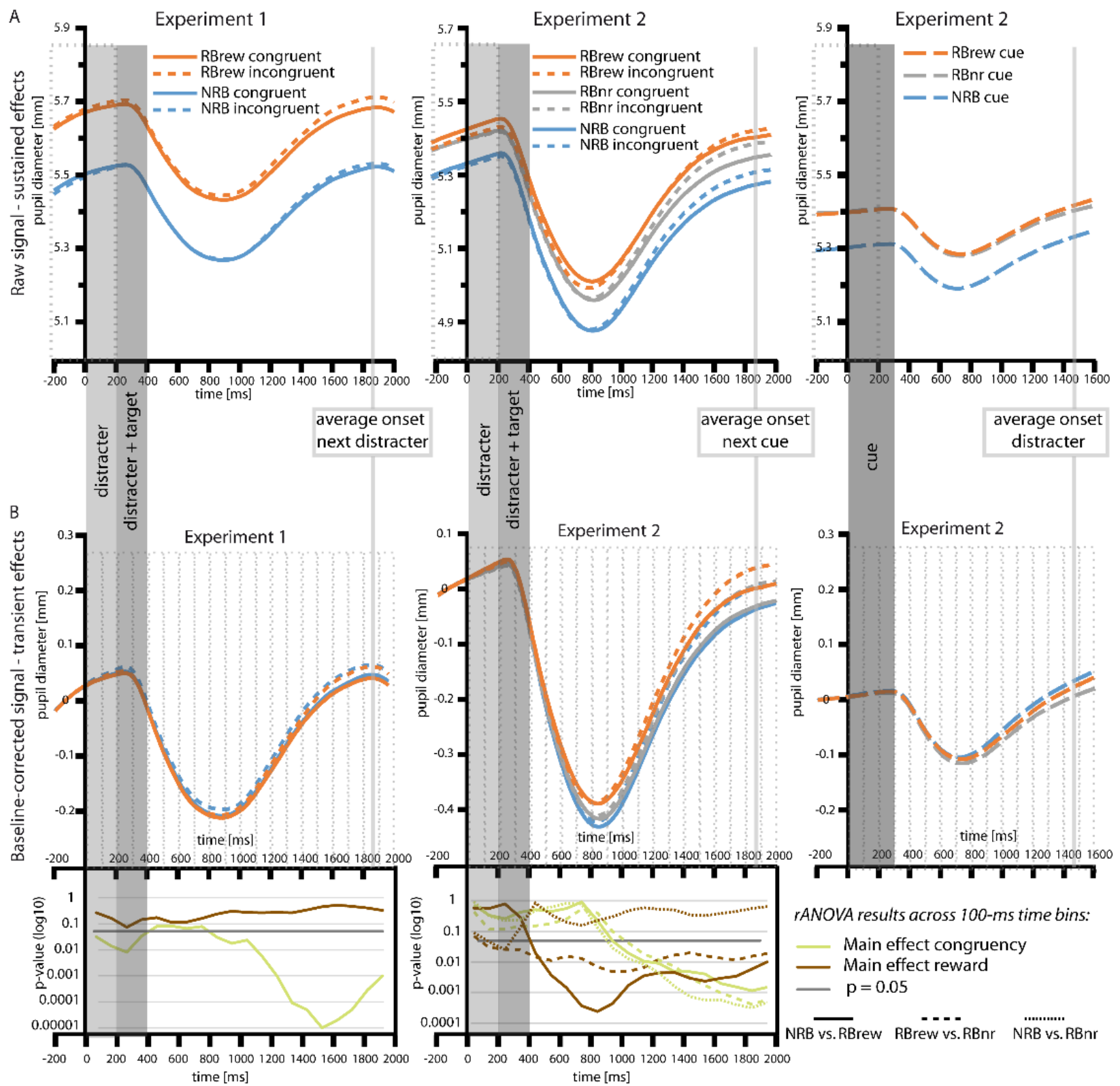

Figure 3. Pupillometry results. A) Sustained effects before baseline correction for target-stimulus in Experiment 1 (left panel), target-stimulus in Experiment 2 (middle panel), and cues in Experiment 2 (right panel). Data was quantified between -200 and $200 \mathrm{~ms}$ (dotted box). B) Transient effects after baseline correction. The inserts at the bottoms display the results of windowed 100-ms analyses (see dotted boxed; $p$-values on a $\log 10$ scale) for a basic 2x2 rANOVA with factors congruency and reward (Experiment 1), and for three separate rANOVAs for Experiment 2 (only main effects of congruency and reward are displayed; the interaction was never significant in either experiment). Uncorrected $p$-values are plotted, but FDR-corrected $p$-values showed qualitatively the same pattern (see main text). Grey vertical bars indicate the timing of events; in addition, the average timing of the subsequent event is displayed. 
Transient effects - cues. In order to look at transient effects on pupil size following cue presentation, we baseline-corrected the data with regard to the respective pre-distracterstimulus period, and again used moving 100 -ms time-windows starting at distracterstimulus presentation. This window ended at $1600 \mathrm{~ms}$, when the light reflex of the subsequent stimulus started to arise. Here, we ran separate paired t-tests for the comparisons of the following trial types: NRB vs. RBrew, RBrew vs. RBnr, and NRB vs. RBnr trials. Throughout the investigated time-range, there were no differences between NRB and RBrew (all uncorrected $p>0.25$; all FDR-corrected $p>0.6$ ), and none between RBrew and RBnr (all uncorrected $p>0.07$; all FDR-corrected $p>0.45$ ). In contrast, tests between 1000 and 1600 ms revealed significant differences between NRB and RBnr (uncorrected $p$-values between 0.002 and 0.03 ; FDR-corrected $p$-values between 0.014 and 0.05 ), signifying wider pupils for the NRB than for the RBnr trials.

Transient effects - targets. For target-stimulus, we again investigated a time-window between distracter-stimulus onset and $2000 \mathrm{~ms}$ in steps of $100 \mathrm{~ms}$. Similar to the behavioral analyses, we did so in three separate two-way rANOVAs with factors congruency and reward, with the latter factor differing across analyses (NRB vs. RBrew, RBrew vs. RBnr, NRB vs. RBnr) to capture different reward-related aspects. None of these analyses ever showed an interaction between congruency and reward (all uncorrected $p>0.25$; all FDR-corrected $p>0.7$ ). The respective results for the main effects are plotted at the bottom middle panel of Figure 3B. This revealed a couple of consistent results. Specifically, a main effect of congruency arose in a rather prototypical fashion starting around $1000 \mathrm{~ms}$ post-stimulus, featuring highly significant results throughout the rest of the time-window (uncorrected $p$-values ranging from 0.0003 to 0.05 ; FDR-corrected $p$-values between 0.002 and 0.09 ). In contrast, the main effect of reward displayed different time-courses for the different analyses. Specifically, when looking for across-block reward effects (NRB vs. RBrew trials, as in Experiment 1), a main effect of reward arose after $400 \mathrm{~ms}$ and stayed significant for the remaining time-window (uncorrected $p$-values between 0.0003 and 0.02 ; FDR-corrected $p$-values between 0.003 and 0.03). The corresponding comparison aimed at the within-block reward effects (RBrew vs. RBnr) yielded an even earlier effect reaching significance already around 100 ms and staying significant until the end of the time-window (uncorrected $p$-values between 
0.005 and 0.03 ; FDR-corrected $p$-values between 0.025 and 0.033 ). Finally, the analysis aimed at across-block context effects (NRB vs. RBnr) seemed to display a short period between 100 and $300 \mathrm{~ms}$ with significant reward effects (uncorrected $p$-values between 0.03 and 0.05 ), which however did not survive FDR correction (all FDR-corrected $p>$ 0.45 ), and also the rest of the time-window displayed the lack of significant effects throughout (all uncorrected other $p>0.09$; all FDR-corrected $p>0.6$ ).

\section{EEG}

\section{Cue-related effects}

Our a-priori interest concerned the cue-related P3 and the subsequent CNV (see Figure 4). Given that congruency is not a factor at this stage yet, we ran three separate pairedsample t-tests to capture the different reward-related aspects. For the P3, this showed significant effects for both comparisons of the RBrew trials with the two different types of non-reward trials (RBrew vs. NRB: $t(21)=3.7 ; p=0.001$; RBrew vs. RBnr: $t(21)=2.9 ; p$ $=0.009)$, but no difference between NRB and RBnr $(p>0.2)$, indicating a P3 increase that was rather exclusive to RBrew trials. The CNV, in turn, showed significant differences for all three comparisons (RBrew vs. NRB: $t(21)=5.5 ; p<0.0001$; RBrew vs. RBnr: $t(21)$ $=2.5 ; p=0.019$; RBnr vs. NRB: $t(21)=3 ; p=0.007)$. This indicates that, in contrast to the P3 effect, the CNV displayed a graded effect across all three conditions (RBrew > RBnr $>$ NRB).
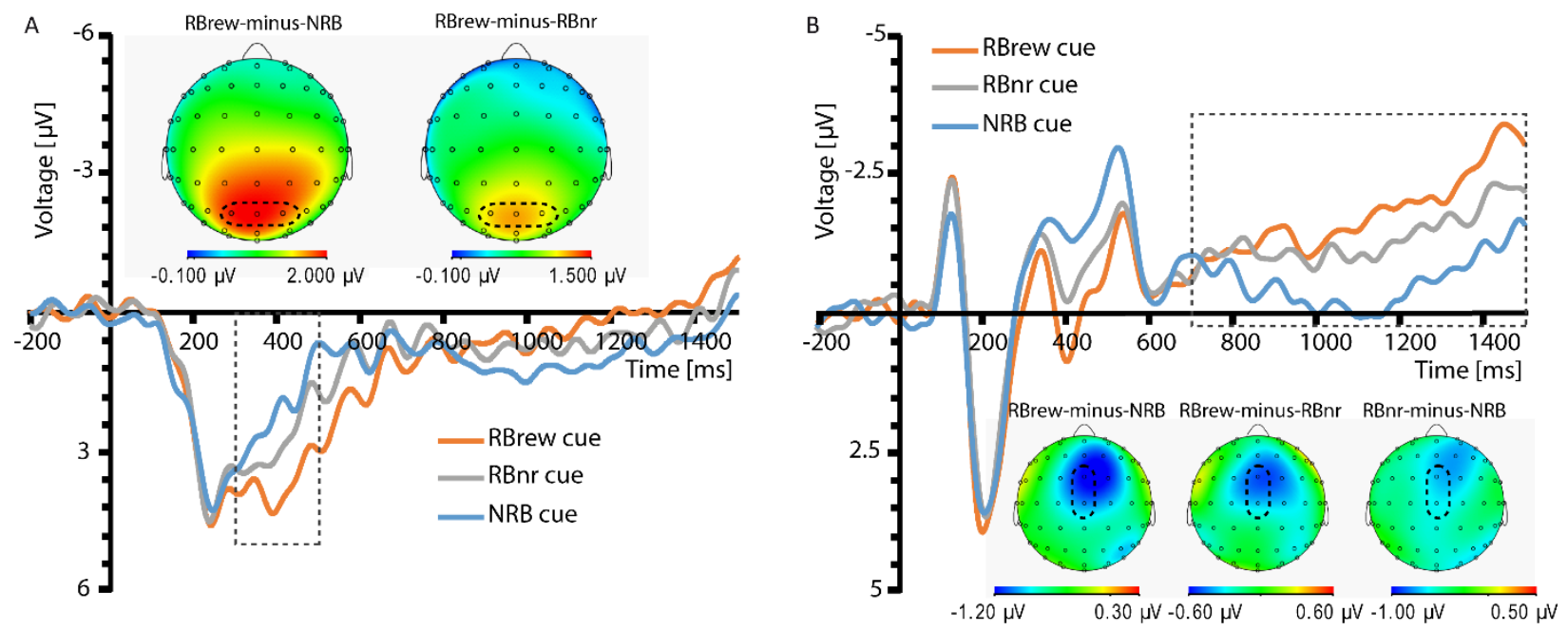

Figure 4. Cue-related effects in Experiment 2. A) P3 results time-locked to cue presentation. B) CNV results time-locked to cue presentation. In both panels, the time- 
course reflects the average data in the ROI highlighted in the topographic maps (dotted elipse). Topographic maps reflect differences across conditions. Note that the scale of those maps differs across plots. The quantified time-range is indicated by a dotted box.

Distracter-related effects $-\mathrm{N} 1$

When performing an analysis corresponding to that of Experiment 1 for the visual $\mathrm{N} 1$ component (see Figure 5B), but split for the three different comparisons possible in Experiment 2, we found a more negative amplitude for both types of trials in reward blocks compared to the non-reward blocks (RBrew vs. NRB: $t(21)=4.1 ; p=0.001$; RBnr vs. NRB: $t(21)=2.6 ; p=0.015)$. In contrast, the two trial types from the reward blocks did not differ significantly (RBrew vs. RBnr: $p>0.1$ ). Note that congruency is not a relevant factor at this point, since it precedes the presentation of the target arrow.

A
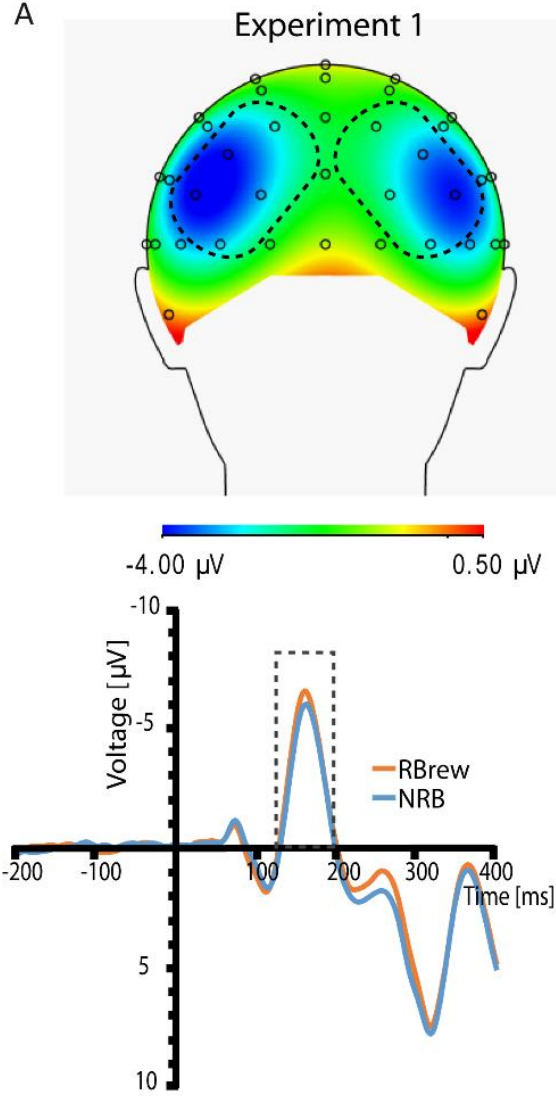

B

Experiment 2
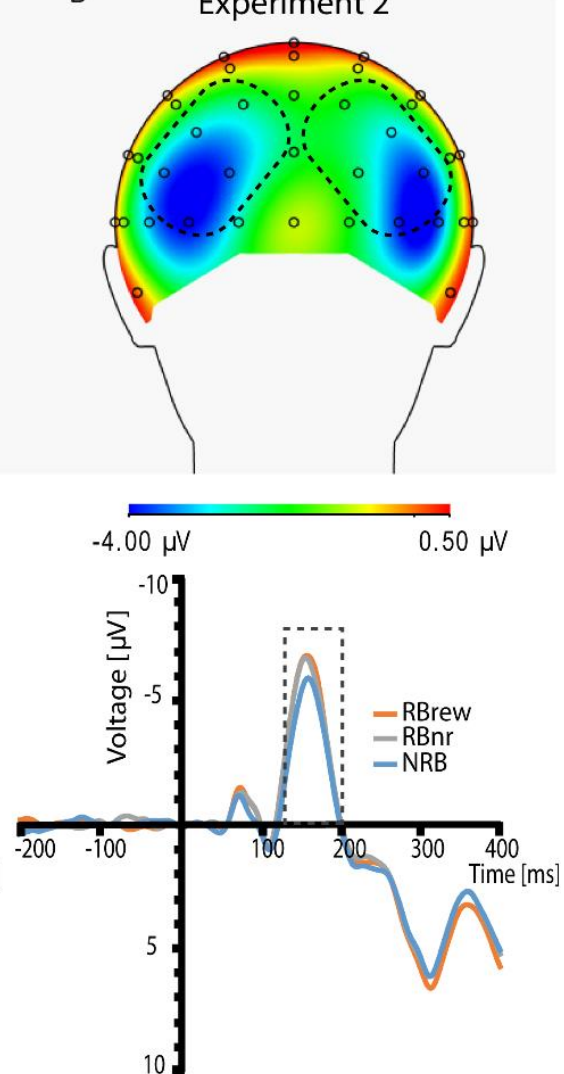

Figure 5. Distracter-related N1 component. A) Experiment 1. B) Experiment 2. In both panels, the time-course reflects the average data in the ROls highlighted in the topographic maps (dotted elipses). The topographic maps reflect the average of conditions. The quantified time-range is indicated by a dotted box. 


\section{Post-target fronto-central reward difference}

We used the same approach as in Experiment 1 to characterize reward differences in the same fronto-central ROI using the three different paired-samples t-tests (see Figure 6, lower three panels). This yielded consistent differences throughout the 300-to-700 ms period for the RBrew vs. NRB comparison (uncorrected $p$-values between 0.0000007 and 0.005; FDR-corrected $p$-values between 0.000006 and 0.005 ) as well as for the RBrew vs. RBnr comparison (uncorrected $p$-values between 0.0001 and 0.02 ; FDR-corrected $p$ values between 0.0009 and 0.02 ). In addition, the RBnr vs. NRB comparison yielded an early difference between 300 and $450 \mathrm{~ms}$ (uncorrected $p$-values between 0.038 and 0.043; FDR-corrected $p=0.05$ ) and a late difference between 500 and $700 \mathrm{~ms}$ (uncorrected $p$-values between 0.0002 and 0.0005 ; FDR-corrected $p$-values between 0.0007 and 0.001$)$.

\section{Baseline Alpha power}

In an equivalent analysis to that in the first experiment, we investigated baseline alpha power to characterize block-level differences in neural activity (Figure 7B/C). For this, we initially turned to the time range preceding the cue-stimulus, again quantifying alpha power in a 500-ms time window. In contrast to the distracter-preceding time window in Experiment 1, we did not observe a block-based difference $(p>0.1)$. Turning to a corresponding analysis of alpha power preceding distracter onset, we did observed a trend-level difference for the RBrew vs. NRB comparison $\left(F(1,20)=4.17 ; p=0.054 ; \eta_{p}^{2}=\right.$ $0.17)$ whereas neither the RBrew vs. RBnr nor the RBnr vs. NRB comparison yielded a significant difference (both $p>0.1$ ). 
A Experiment 1 - across-block reward effect (RBrew-minus-NRB)

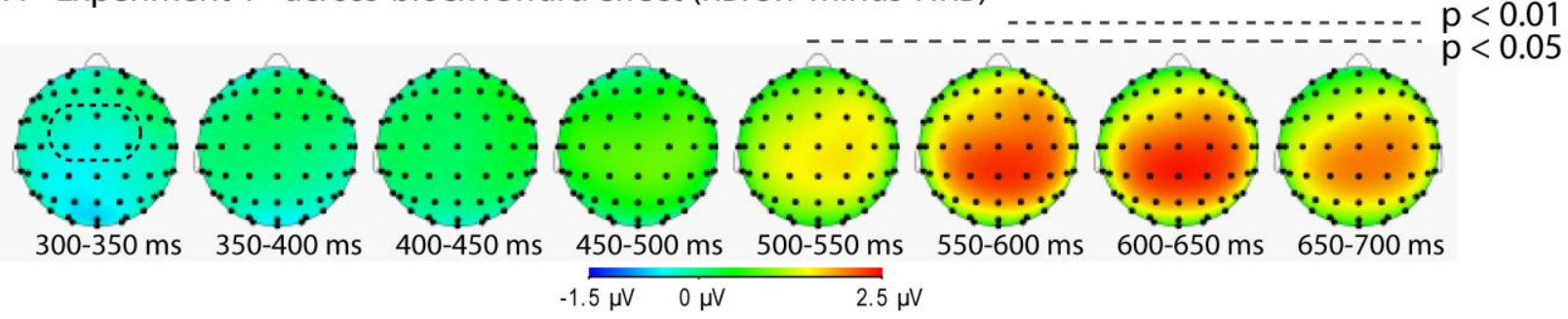

B Experiment 2 -across-block reward effect (RBrew-minus-NRB) $p<0.001$

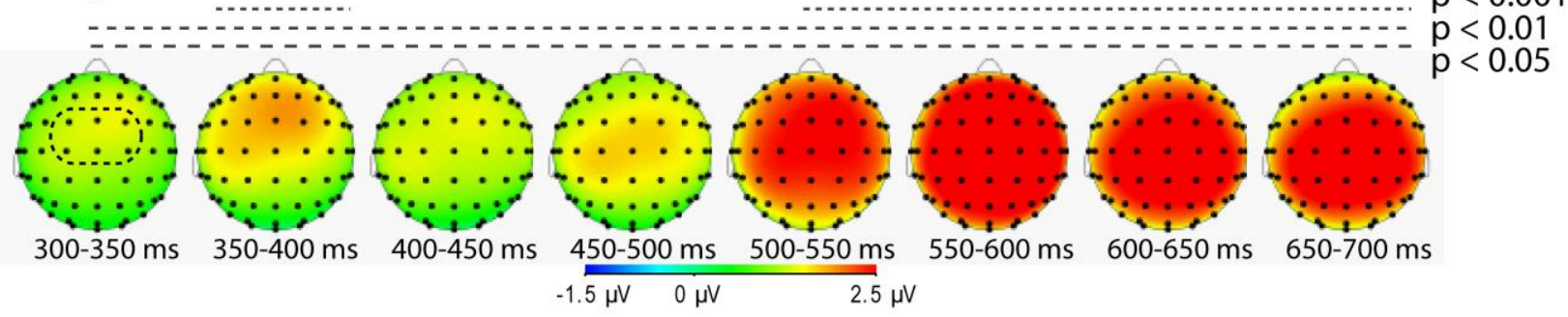

Experiment 2 - within-block reward effect (RBrew-minus-RBnr) $p<0.001$

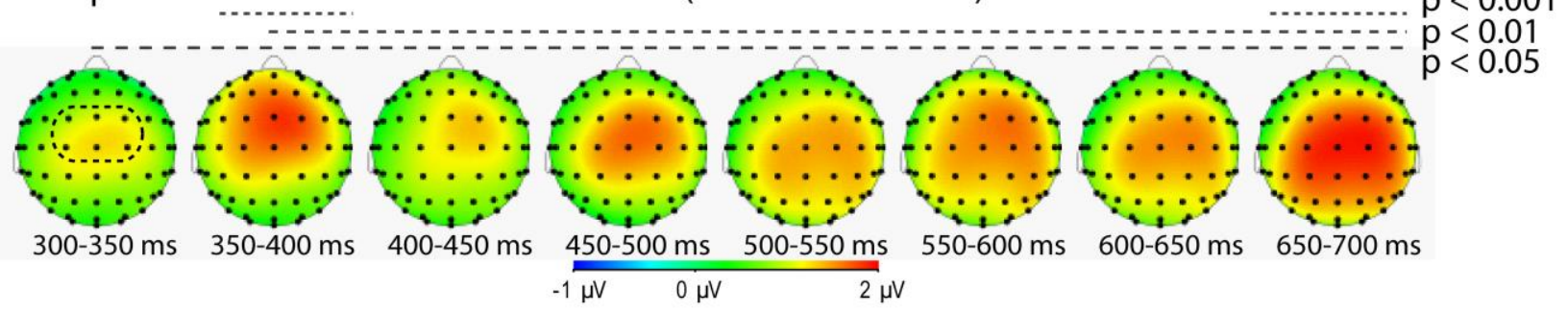

Experiment 2 - across-block context effects (RBnr-minus-NRB) $\mathrm{p}<0.001$

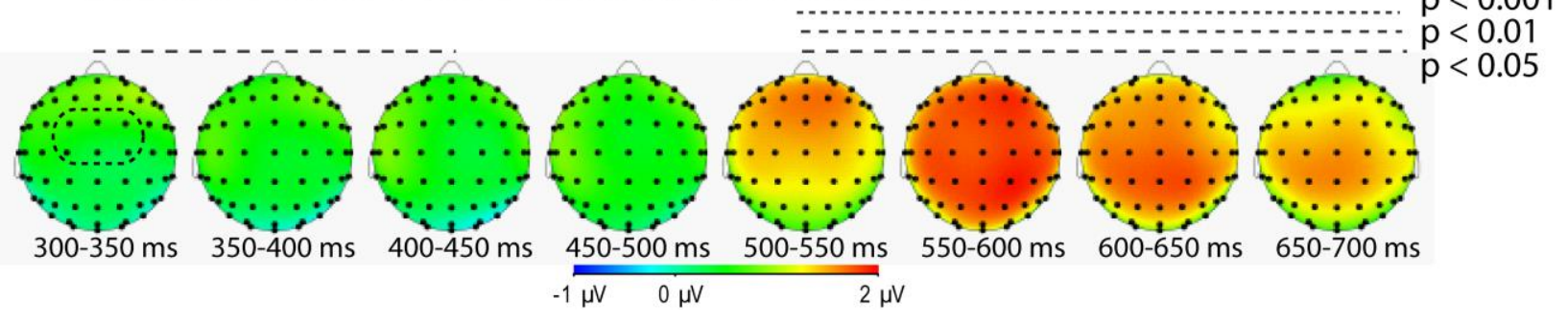

Figure 6. Fronto-central reward-related differences. A) Experiment 1. B) Experiment 2. Throughout the figure, dotted lines above the maps indicate the significance level of the respective reward comparison. Note that the scale of the topographic maps differs across different plots. Uncorrected p-values are plotted, but FDR-corrected p-values showed qualitatively the same pattern (see main text). 

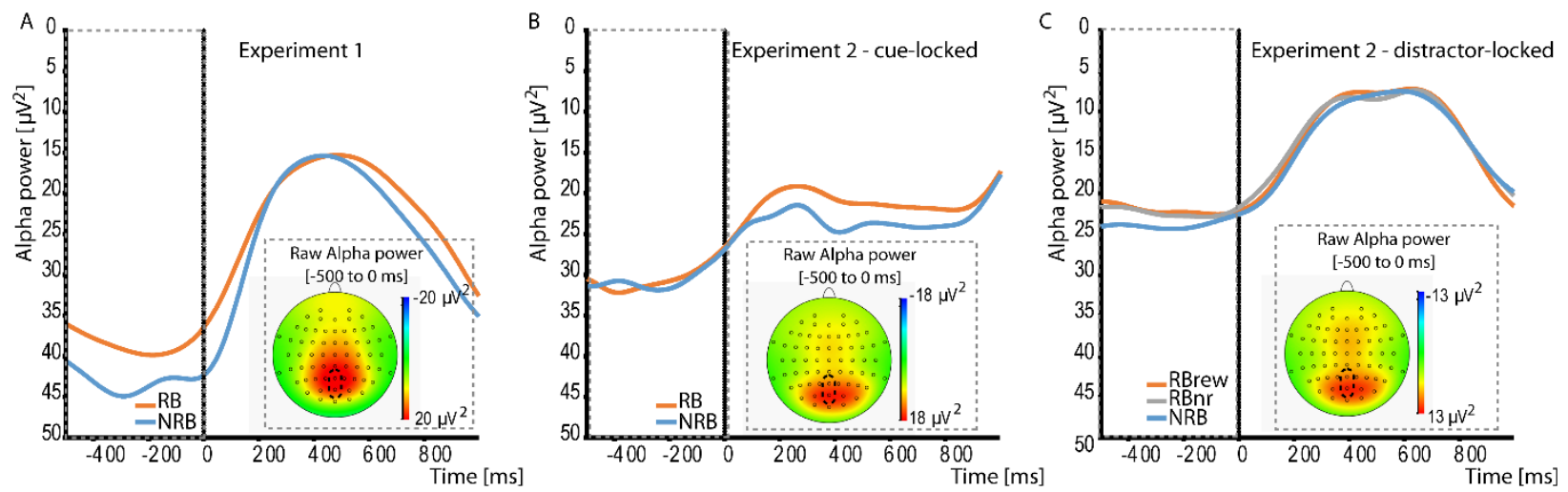

Figure 7. Baseline Alpha power. A) Experiment 1. B) Experiment 2 cue-locked. C) Experiment 2 distracter-locked. Differential alpha power is displayed as the average signal of $\mathrm{Pz}$ and $\mathrm{POz}$ (see ellipse in topographic maps for location). The inserts show topographic distributions of the average of alpha power in the 500-ms baseline period (see dashed boxes) across the respective conditions, illustrating that the chosen ROI covered the raw alpha-power maximum in all conditions.

\section{BETWEEN-EXPERIMENT COMPARISONS}

\section{Behavior}

We directly compared the behavioral results from the two experiments. Given power considerations in this between-subject context, and the fact that there were no differences between the different no-reward trials (NRB vs. RBnr), we averaged those data and compared them with the reward-related trials (RBrew) in a two-by-two rANOVA with the additional between-subject factor Experiment. This analysis confirmed that in Experiment 1 , participants responded faster in general $\left(F(1,42)=4.83 ; p=0.034 ; \eta_{p}^{2}=0.1\right)$, which went along with globally reduced accuracy $\left(F(1,42)=6.62 ; p=0.014 ; \eta_{p}^{2}=0.14\right)$. Importantly, the reward effect on RT was indeed larger in Experiment 2 than (the absent effect) in Experiment $1\left(F(1,42)=4.35 ; p=0.043 ; \eta_{p}^{2}=0.09\right)$.

\section{Pupil size}

Sustained effects. In parallel to the behavioral data analysis, we again collapsed across the two types of no-reward trials (NRB and RBnr) in order to increase power and decrease complexity given that there was no clear difference between these conditions. We then performed a 2x2 rANOVA with the within-subject factors reward and congruency, and the additional between-subject factor experiment. For sustained effects, this analysis 
furthermore included the factor of block-order as a covariate. Comparing the targetstimulus time-range across experiments, this analysis did not reveal a significant interaction with experiment for the main effects of reward, congruency, or their interaction (all $p>0.1$ ), indicating comparable modulations of sustained pupil size by reward.

Transient effects. For transient effects, again analyzed in an equivalent fashion across 100-ms windows and collapsing across NRB and RBnr trials, results indicated early interactions between experiment and congruency (between 0 and $600 \mathrm{~ms}$ for uncorrected $p$-values between 0.003 and 0.037 , and between 100 and $300 \mathrm{~ms}$ for FDR-corrected $p=$ 0.03). More importantly, experiment interacted with reward throughout the entire timewindow with the exception of the first 100 window (100-2000 ms: uncorrected $p$-values between 0.0001 and 0.032 and FDR-corrected $p$-values between 0.001 and 0.034 ).

\section{EEG}

Distracter-related effects - N1. For this comparison, we collapsed across the two types of reward trials in Experiment 2 (RBrew and RBnr) that did not differ, and performed two independent-samples t-tests comparing the resulting conditions in the RB block and NRB block with their counterparts in Experiment 1. Despite the fact that the N1 amplitudes were numerically larger in Experiment 1 then in Experiment 2, the respective differences across experiments did not reach statistical significant (both $p>0.5$ ).

Post-target fronto-central reward difference. Given the differences also between the RB conditions, we did not collapse across conditions for the data of Experiment 2, and limited ourselves to the same between-block comparison in both experiments. To this end, we ran independent-samples t-tests for the resulting across-block reward differences (RBrew-minus-NRB) to compare them across experiments. This analysis yielded significant differences across the two experiment between 300 and $450 \mathrm{~ms}$ ( $p$-values between 0.008 and 0.019 ; FDR-corrected $p=0.05$ ), indicating that there was a stronger reward-related fronto-central difference in Experiment 2 than in Experiment 1 in this early time-range.

Baseline alpha power. When comparing the RB vs. NRB block differences in baseline alpha power across experiments, we failed to observe significant differences both when 
comparing the pre-distracter data from Experiment 1 with the corresponding pre-distracter data from Experiment 2, as well as when comparing the pre-distracter time range from Experiment 1 with the pre-cue time-range of Experiment 2 (both $p>0.1$ ).

\section{Discussion}

In the present report, we compared data from two experiments that associated reward in different fashions with a Flanker task, investigating markers of effort to characterize its role in bringing about reward-related behavioral benefits. Specifically, we used a pure block-based reward manipulation in Experiment 1, with the whole series of targets in a block either being reward-related or not. In Experiment 2 we used trial-based cues, while still maintaining the general block structure with one block type containing exclusively noreward trials, and the other block type containing reward-related and no-reward trials. Experiment 1 yielded no behavioral reward benefit, whereas Experiment 2 did. We found increased sustained pupil size in both experiments for the reward-related blocks, with an additional transient reward-related increase in pupil size only in Experiment 2. Additionally comparing the EEG data from the two experiments, the clearest difference was a frontocentral reward-related difference that arose earlier in the second experiment, likely reflecting reward modulations of target selection. The present data therefore converge with earlier work suggesting that reward triggers a more proactive control state involving enhanced preparatory effort, and indicate that in the present task context a transient form of preparatory effort was more relevant for behavior than a sustained form.

\section{Distinguishing sustained and transient pupil size as markers of effort}

Pupil size has long been considered as a rather specific correlate of mental effort (Beatty, 1982b; Kahneman \& Beatty, 1966), and when it scales with task difficulty, this is assumed to reflect recruitment of effort in response to such task demands (e.g., Boehler, et al., 2011). In addition, it has been shown to reflect both transient (Alnaes, et al., 2014; Laeng, et al., 2011) and sustained increases in effort (Cabestrero, Crespo, \& Quiros, 2009; Massar, et al., 2016), as well as their co-existence (Chiew \& Braver, 2013). Moreover, empirical data from a task context also involving a reward-based incentive scheme suggest that pupil size indeed reflects more closely the effort triggered by reward prospect 
than the reward value itself (Massar, et al., 2016), although others have argued in a comparable context that it might partly also reflect value information and their more affective representation (Chiew \& Braver, 2013). Given the fact that reward also has an affective dimension (Notebaert \& Braem, 2015), a clear distinction of effort-related and affect-related aspects is not easily possible in reward-related tasks like the present ones. Yet, it is important to note that the present tasks do not strongly emphasize the affective dimension of reward manipulations, in that reward feedback is only given intermittently, and that neither targets nor cues are not directly linked to reward (as would be, e.g., cues presented as dollar signs).

In the present experiments, we found a dissociation between reward modulations of sustained vs. transient effects. Specifically, Experiment 1 featured large sustained differences in pupil size that were comparable in size to a recent study that linked sustained effort-related increases in pupil size to increased performance in a vigilance task (Massar, et al., 2016). Yet, in contrast to that study, our results in Experiment 1 did not show any signs of a behavioral benefit despite the sizeable sustained pupil size difference between the reward-related and the no-reward block. Such a sustained effect of pupil size was also observed in our Experiment 2. Importantly, however, this experiment furthermore showed clear transient reward effects on pupil size as well (see also Chiew \& Braver, 2013), which jointly seems to suggest that the transient pupil response reflects a process that is more relevant for task performance in the present task. This notion is in fact consistent with classic work on pupil size and auditory vigilance performance that has also found that transient responses in pupil size were linked to behavioral performance, whereas sustained pupil size was not (Beatty, 1982a). Yet, whether or not sustained pupil size is related to performance might also be a function of the investigated task, with recent data, for example, showing a tight relationship between slow fluctuations in pupil size, brain network connectivity states, and task performance in an n-back task (Shine, et al., 2016). Still, given the frequent co-occurrence of sustained and transient pupil-size modulations, which are not always disentangled (Massar, et al., 2016), it is not always clear in how far transient pupil-size modulations might not also be involved in contexts that are mainly characterized by sustained pupil-size differences. 
Pupil size has traditionally been linked to activity in the norepinephrinergic system (for a review, see Eckstein, et al., 2017; but, see also Reimer, et al., 2016 for a possible dissociation between this link for transient and sustained pupil size), with phasic and tonic firing modes of this system being reflected in transient vs. sustained pupil size (AstonJones \& Cohen, 2005; Gilzenrat, Nieuwenhuis, Jepma, \& Cohen, 2010; Murphy, O'connell, O'sullivan, Robertson, \& Balsters, 2014; Murphy, Robertson, Balsters, \& O'Connell $R, 2011$ ). While a joint increase in sustained and transient pupil-size as in the present Experiment 2 might not be fully consistent with theoretical accounts that often suggest an inverse relationship (Aston-Jones \& Cohen, 2005), the present results are similar to an earlier strongly related report that used a reward manipulation in an $A X$ continuous performance task (Chiew \& Braver, 2013). What seems more important, in the present context, however, is the dissociation across experiments, where Experiment 2 featured increases in both sustained and transient pupil size related to reward, whereas Experiment 1 only showed the former.

In the context of cognitive effort and pupil size, it is interesting that pupil size has been linked most clearly to the NE system, whereas accounts of cognitive effort usually emphasize the role of the dopaminergic (DA) system (Shenhav, et al., 2017; Westbrook \& Braver, 2016). At the same time, the NE and DA system are highly interrelated in their function (for a review, see Xing, Li, \& Gao, 2016), with recent data suggesting dissociable but related functions in the context of effort (Varazzani, San-Galli, Gilardeau, \& Bouret, 2015). Hence, the apparent disconnect between the concepts of cognitive effort, pupil size and the NE and DA systems might in fact be overemphasized, but rather point to the cooperative functioning of these two key neuromodulator systems in cognitive effort, which future research should further disentangle (see also Chiew \& Braver, 2013).

A striking feature of the transient reward effects on the pupil in Experiment 2 was their latency, starting more or less simultaneously with target presentation when comparing reward-related trials with the no-reward trials from the same blocks, and after approximately $400 \mathrm{~ms}$ when compared with the no-reward trials from the no-reward blocks. This contrasts with the typical latency of (lighting-independent) trial-based pupilsize effects as well as with that of the congruency effects in the present work. The latter started to arise (consistently and strongly) around $1000 \mathrm{~ms}$ after stimulus onset, which is 
very similar to earlier work (Laeng, et al., 2011). In this context it is critical to realize that the relevant information for congruency was only available at target presentation, whereas all relevant information about reward was available already at cue presentation, and hence approximately 1.5 seconds before stimulus onset. Although the cue-locked analysis did not show strong transient effects of this kind yet, the target-locked effects are clearly related to this reward-related cue information, and we assume that at least the early portions of the transient pupil-size differences in the target time-range still relate more strongly to the cue information than to the processing of the target. As we will argue below, we believe that the temporal set-up of Experiment 2 favors transient increases in preparatory effort by reward, which in turn might be critical in bringing about behavioral benefits in the present task context.

\section{Transient preparatory effort reflected in the EEG}

Since we believe that the behavioral reward benefit and the transient reward-related modulation of pupil size in Experiment 2 both relate to transient preparatory effort triggered by the reward cues, it was important to further corroborate the presence of such processes. For this, we turned to the EEG data, and in particular the P3 and CNV components elicited by the cue-stimuli. P3 modulations are typical in reward tasks and likely reflect the registration of and orientation to this relevant information (e.g., Goldstein, et al., 2006; Hughes, Mathan, \& Yeung, 2013; Schevernels, et al., 2014). Importantly in the present context, the P3 was only clearly enhanced for the actually reward-related trials, suggesting that this component reflected the registration of reward prospect.

Turning to the CNV, classic work has linked it to enhanced task preparation (Tecce, 1972), and it has been explicitly linked to preparatory activity in fronto-parietal control areas (Grent-'t-Jong \& Woldorff, 2007). Reward-related enhancements of the CNV have been reported before, and usually go along with a behavioral benefit in the subsequent task (Schevernels, et al., 2014; van den Berg, et al., 2014). The study by van den Berg et al. (2014), in particular, is closely related to the present Experiment 2, in that it combined reward-predictive cues (vs. no-reward cues, and control cues) with a Stroop task. Those authors also observed clear enhancements of the CNV for reward, and furthermore found a specific relationship to subsequent response speed. 
Effort is often conceptualized, at least in part, in a way that is related to enhanced cognitive control, likely in a manner in which the dopaminergic system plays key roles (Braver \& Cohen, 2000; Braver, Gray, \& Burgess, 2007; Westbrook \& Braver, 2015, 2016). Crucially, it seems that incentives can influence this process and lead to performance enhancements based on greater cognitive control (Botvinick \& Braver, 2015; Notebaert \& Braem, 2015), in particular by shifting to a (more effortful) proactive control mode (Braver, 2012). In this regard, the enhanced CNV in the present Experiment 2 (and earlier related work) fits very well, since it is not just believed to reflect such control processes, but because it has also been suggested to be related to activity in the dopaminergic system (Linssen, et al., 2011). Due to its non-immediate mode of operation (Seamans \& Yang, 2004), dopaminergic effects would not be expected to arise instantaneously. Consistent with this, it has recently been discussed, how reward-cue information would take a couple of hundreds of milliseconds in order to take an effect (Chiew, Stanek, \& Adcock, 2016; but see also Janssens, De Loof, Pourtois, \& Verguts, 2016), as was possible in the present Experiment 2.

In this context of task preparation, the absence of an interaction between reward and congruency in the present experiments is relevant (concerning behavioral results and the majority of pupillometry and EEG results). Such interactions are sometimes found in comparable contexts (e.g., Krebs, et al., 2013; Krebs, Boehler, \& Woldorff, 2010; Padmala \& Pessoa, 2011), but not always (e.g., Krebs, et al., 2011; van den Berg, et al., 2014). It seems likely that this depends on specific task features, related to what kind of information can be enhanced or inhibited in order to specifically reduce the size of the incongruency effect under reward (discussed in van den Berg, et al., 2014). In a more global context, however, and with reference to enhanced proactive control and preparatory effort, it seems quite likely that an interaction between reward and congruency could arise if both aspects (reward availability and congruency) would be cued, rather than just reward, as has been shown before (Chiew \& Braver, 2016).

Finally, we investigated block-level differences in alpha power preceding the taskrelevant stimuli in the two experiments. Alpha power is generally linked to vigilance and attention, with lower amplitudes signifying more attentive states (Cajochen, et al., 1995). Importantly, in order to characterize event-independent modulations, we did not baseline- 
correct the data. Based on this data, we were interested to find possible electrophysiological correlates of the sustained pupil size effect that was consistently present in both experiments, and in all phases of the second experiment. This was clearly the case in Experiment 1, where we observed a strong attenuation of alpha power preceding targets in the reward-related compared to the no-reward blocks. Yet, counter to the clear presence of sustained pupil-size modulations by reward also in both trial phases in Experiment 2, we observed only equivocal evidence concerning concomitant alpha power modulations. Specifically, we did not observe such modulations preceding cue-stimuli, but a (trend-level) effect preceding target-stimuli. One could speculate that reward led to a more transient form of preparatory effort in Experiment 2 than in Experiment 1. Furthermore, the result pattern seems to hint at a dissociation between processes related to sustained pupil size (increased by reward throughout both experiments) and alpha power (most clearly modulated in Experiment 1). Yet, it is important to realize that the present analysis of alpha power was rather post-hoc (modeled on the sustained pupil size analysis), and that a direct comparison across experiments did not yield strong support for a quantitative difference. More work is therefore needed to explore the role of block-level alpha power modulations by reward and their relationship to sustained pupil size.

\section{Implementation of a behavioral benefit during target processing}

In the current experiment, we used a Flanker task to present distracter arrows consistently $200 \mathrm{~ms}$ before the target arrows, which was originally intended to specifically look exclusively at effects of distracter processing in the first $200 \mathrm{~ms}$ dissociated from later target processing (Appelbaum, et al., 2012). We hypothesized that in a reward context, less attention would be allocated to the distracter arrows, reflected by a decreased attention-related N1 component (Hopf, Vogel, Woodman, Heinze, \& Luck, 2002; Vogel \& Luck, 2000). A decrease in attentional allocation was expected based on previous work of ours that showed such strategic down-regulation of attention in a conflict-adaptation context (Bombeke, et al., 2017).

Yet, the resulting data pattern was more complicated. Specifically, Experiment 1 did not show any clear modulations of this early attentional component by reward. 
Experiment 2, in turn, did, but this was dominated by a block effect, with similarly enhanced amplitudes for both trial types in the reward blocks. Hence, the modulation was in fact even going in the opposite direction of what we expected. While this is interesting from the standpoint of a context effect (Jimura, et al., 2010), it clearly does not map onto the behavioral results. Rather, it might represent a within-trial context effect reflecting the inability to temporarily down-regulate the processing of a given stimulus that is not taskrelevant when it is rapidly followed by a task-relevant one, which one would want to attend to particularly strongly (Langford, Schevernels, \& Boehler, 2016; Schevernels, et al., 2015).

An additional context effect that is important in the mixed block-event-related reward design implemented in Experiment 2 refers to differences between no-reward trials from the no-reward vs. from the reward block. Specifically, the latter have been shown "profit" from the context of reward trials in different task contexts, which was interpreted as indicating increased sustained proactive control in reward contexts (Chiew \& Braver, 2013; Jimura, et al., 2010). In this regard, the present data were slightly inconclusive. Behaviorally, there was no indication of such a block-level context effect, and also the transient pupil response showed no such effect. In contrast, the EEG data indicated a reward-context effect on the fronto-central P3-like component. Specifically, it was more pronounced for RBnr trials than for NRB trials, which, however, apparently had no effect on behavior. As such, it is interesting to note that the latency of the pronounced part of this effect was comparable to that of the block difference in Experiment 1, which also lacked a behavioral reward effect. This raises the possibility that in Experiment 2, a block-level reward context effect merely did not manifest behaviorally potential due to task dynamics (e.g., the overall high response speed). Still, taking the absent behavioral context effect at face value, it is worth speculating about differences of reward association to a task, and whether such differences could drive the presence or absence of blocklevel reward context effects. Among the many features that can vary between different experiments investigating reward (Bonner, et al., 2000; Dambacher, Hubner, \& Schlosser, 2011; Krebs \& Woldorff, 2017), the type of reward feedback stands out as a clear difference between the present and earlier studies. Specifically, in the present work, as well as in other work where we did not find clear indications of reward context effects 
(Krebs, et al., 2010; Schevernels, et al., 2015), we have used cumulative feedback, whereas studies that found such context effects usually provide trial-level feedback (Chiew \& Braver, 2013; Jimura, et al., 2010). Given the important theoretical role that context effects play concerning reward modulations of proactive control (Braver, 2012), it will be important going forward to investigate which factors determine whether they arise or not.

Turning to the time-window after the target arrows were presented, in accordance with earlier related work (Krebs, et al., 2013; van den Berg, et al., 2014), we also explored later modulations that are likely still related to attentional processes, and in this case probably with target selection. Specifically, the reward-minus-no-reward difference in Experiment 1 yielded a positive modulation over fronto-central sites resulting from a stronger positive deflection in reward-related trials, very similar to the respective difference described by van den Berg et al. (2014), albeit a bit later. In the present Experiment 2, however, the similarity to the results of van den Berg et al. (2014) even extended to the latency and duration of the effect. Importantly, that was the case when comparing reward-related trials both to no-reward trials within the reward-related blocks and to trials from the no-reward blocks. This P3-like (maybe specifically P3a-like) component likely reflects enhanced processing of the targets under reward (Goldstein, et al., 2006; Polich, 2007). The key aspect here is that this difference arose much earlier in Experiment 2 than in Experiment 1, suggesting that it is only relevant to behavior if it arises fast enough. Finally, there was a similar modulation also for the comparison of different no-reward trials from the two block types, with larger amplitudes for no-reward trials from reward blocks, but again relatively late. While again representing a potentially interesting context effect, it clearly did not affect behavior.

\section{The absence of a behavioral benefit in a pure block reward context}

It is important to contextualize the absence of a behavioral benefit in the pure block-based context of Experiment 1. While maybe not as consistently as cueing-based approaches, block-based reward schemes are nevertheless also known to bring about behavioral benefits (e.g., Kouneiher, et al., 2009; Locke \& Braver, 2008; Massar, et al., 2016; Paschke, et al., 2015). Importantly, such effects could in fact rely on similar mechanisms as characterized in the present Experiment 2. Specifically, even under the notion that 
transient preparatory increases of effort would be needed to achieve a behavioral benefit, participants could still have tried to specifically prepare for all trials in the reward-related block of Experiment 1 based on the relatively regular trial timing (although the inter-target interval was a bit longer than the cue-target interval). Interestingly, this did not seem to be the case. In addition, it is important to note that in transforming the logic of Experiment 1 into a classic cuing experiment for Experiment 2, we additionally included no-reward trials, as is customary. Naturally, the presence of no-reward trials could have accentuated the actual reward-related trials, and it seems possible that their absence in Experiment 1 might have contributed to the absent behavioral benefit. Yet, even if one assumes that dropping the no-reward trials from the reward-related blocks in Experiment 2 could have abolished the behavioral reward effect, we would expect that it would simultaneously abolish the transient preparatory effort effects found in Experiment 2, which we consider the main result of the present work.

When considering the differences between Experiments 1 and 2, it is obvious that participants in Experiment 2 had a slightly more conservative speed-accuracy tradeoff (for a relevant discussion, see Chiew \& Braver, 2011). It generally seems possible that faster performance in Experiment 1 relates to sustained performance that is not interrupted and/or accentuated by other events like cues or feedback. This contrasts with the design of Experiment 2, where cue-stimuli constantly interrupted the sequence of targets and allowed for reward-trial-specific preparation. Although some studies have started to investigate the effect of reward on speed-accuracy tradeoffs (e.g., Dambacher, et al., 2011; Hubner \& Schlosser, 2010), a fuller understanding of which factors drive such differences in speed-accuracy tradeoffs is still needed. At the same time, given the numerous aspects that differed meaningfully between the different conditions in Experiment 2 (and in comparison with Experiment 1), pertaining in particular markers of transient preparatory effort and attentional selection, we do not think that the present reward benefit is solely a function of a speed-accuracy tradeoff. In addition, it is important to stress that participants were instructed the same way in both experiments, so that one might rather consider the speed-accuracy tradeoff differences as an outcome variable of the experiments. 


\section{Conclusion}

In summary, the present data suggest that behavioral reward effects likely depend on whether or not participants succeed in ramping up preparatory effort ahead of the task. As such, the present work strongly converges with prominent theoretical models that suggest that reward triggers enhanced effortful proactive control (Braver, 2012; Braver, et al., 2007; Westbrook \& Braver, 2016), which have already received significant empirical support (e.g., Braver, Paxton, Locke, \& Barch, 2009; Chiew \& Braver, 2013; Jimura, et al., 2010). The present data seem to add that under certain task contexts like the present one, transient increases in preparatory effort are more relevant for achieving a behavioral benefit than sustained ones.

Going forward, it will be important to investigate in how far these relationships remain intact under various task and reward regimes. In addition, future work should attempt to directly link the pupillometry and EEG data, which has significant potential to further understand the relationship of the underlying processes (Eckstein, et al., 2017). To our knowledge, only very few studies have thus far attempted to directly integrate data from these modalities through covariational within-subject analyses (Hong, et al., 2014; Murphy, et al., 2011; see also Wolff, Muckschel, Ziemssen, \& Beste, 2018). Interestingly from the perspective of the current data, this work has related variations of sustained pupil size with fronto-central P3 modulations, both of which were individually modulated in the present work. Given their promising results, as well as the power of single-trial-oriented covariational approaches (Hubner \& Schlosser, 2010; Pernet, Chauveau, Gaspar, \& Rousselet, 2011), a further integration of these complimentary data modalities holds significant promise for better understanding the relationship between pupil size, motivation, and effort in the future. 


\section{Bibliography}

Alnaes, D., Sneve, M. H., Espeseth, T., Endestad, T., van de Pavert, S. H., \& Laeng, B. (2014). Pupil size signals mental effort deployed during multiple object tracking and predicts brain activity in the dorsal attention network and the locus coeruleus. J Vis, 14.

Appelbaum, L. G., Boehler, C. N., Won, R., Davis, L., \& Woldorff, M. G. (2012). Strategic allocation of attention reduces temporally predictable stimulus conflict. $J$ Cogn Neurosci, 24, 1834-1848.

Aston-Jones, G., \& Cohen, J. D. (2005). An integrative theory of locus coeruleusnorepinephrine function: adaptive gain and optimal performance. Annu Rev Neurosci, 28, 403-450.

Babiloni, C., Brancucci, A., Vecchio, F., Arendt-Nielsen, L., Chen, A. C., \& Rossini, P. M. (2006). Anticipation of somatosensory and motor events increases centroparietal functional coupling: An EEG coherence study. Clin Neurophysiol.

Beatty, J. (1982a). Phasic not tonic pupillary responses vary with auditory vigilance performance. Psychophysiology, 19, 167-172.

Beatty, J. (1982b). Task-evoked pupillary responses, processing load, and the structure of processing resources. Psychological Bulletin, 91, 276.

Benjamini, Y., \& Hochberg, Y. (1995). Controlling the false discovery rate: a practical and powerful approach to multiple testing. Journal of the royal statistical society. Series B (Methodological), 289-300.

Boehler, C. N., Hopf, J. M., Krebs, R. M., Stoppel, C. M., Schoenfeld, M. A., Heinze, H. J., \& Noesselt, T. (2011). Task-Load-Dependent Activation of Dopaminergic Midbrain Areas in the Absence of Reward. J Neurosci, 31, 4955-4961.

Bombeke, K., Langford, Z. D., Notebaert, W., \& Boehler, C. N. (2017). The role of temporal predictability for early attentional adjustments after conflict. PLoS One, 12, e0175694.

Bonner, S. E., Hastie, R., Sprinkle, G. B., \& Young, S. M. (2000). A review of the effects of financial incentives on performance in laboratory tasks: Implications for management accounting. Journal of Management Accounting Research, 12, 1964.

Botvinick, M., \& Braver, T. (2015). Motivation and cognitive control: from behavior to neural mechanism. Annu Rev Psychol, 66, 83-113.

Bradley, M. M., Miccoli, L., Escrig, M. A., \& Lang, P. J. (2008). The pupil as a measure of emotional arousal and autonomic activation. Psychophysiology, 45, 602-607.

Braver, T. S. (2012). The variable nature of cognitive control: a dual mechanisms framework. Trends Cogn Sci, 16, 106-113.

Braver, T. S., \& Cohen, J. D. (2000). On the control of control: the role of dopamine in regulating prefrontal function and working memory. In S. Monsell \& J. Driver (Eds.), Attention and Performance XVIII (pp. 713-738). Cambridge, MA: MIT Press.

Braver, T. S., Gray, J. R., \& Burgess, G. C. (2007). Explaining the Many Varieties of Working Memory Variation: Dual Mechanisms of Cognitive Control. In A. R. A. Conway, C. Jarrold, M. J. Kane, A. Miyake \& J. N. Towse (Eds.), Variation in Working Memory (pp. 76-106). Oxford Oxford Univerity Press. 
Braver, T. S., Paxton, J. L., Locke, H. S., \& Barch, D. M. (2009). Flexible neural mechanisms of cognitive control within human prefrontal cortex. PNAS.

Cabestrero, R., Crespo, A., \& Quiros, P. (2009). Pupillary dilation as an index of task demands. Percept Mot Skills, 109, 664-678.

Cajochen, C., Brunner, D. P., Krauchi, K., Graw, P., \& Wirz-Justice, A. (1995). Power density in theta/alpha frequencies of the waking EEG progressively increases during sustained wakefulness. Sleep, 18, 890-894.

Camerer, C. F., \& Hogarth, R. M. (1999). The effects of financial incentives in experiments: A review and capital-labor-production framework. Journal of risk and uncertainty, 19, 7-42.

Chelazzi, L., Perlato, A., Santandrea, E., \& Della Libera, C. (2013). Rewards teach visual selective attention. Vision Res, 85, 58-72.

Chiew, K. S., \& Braver, T. S. (2011). Monetary Incentives Improve Performance, Sometimes: Speed and Accuracy Matter, and so Might Preparation. Front Psychol, 2, 325.

Chiew, K. S., \& Braver, T. S. (2013). Temporal dynamics of motivation-cognitive control interactions revealed by high-resolution pupillometry. Front Psychol, 4, 15.

Chiew, K. S., \& Braver, T. S. (2016). Reward favors the prepared: Incentive and taskinformative cues interact to enhance attentional control. Journal of Experimental Psychology: Human Perception and Performance, 42, 52.

Chiew, K. S., Stanek, J. K., \& Adcock, R. A. (2016). Reward Anticipation Dynamics during Cognitive Control and Episodic Encoding: Implications for Dopamine. Front Hum Neurosci, 10.

Dambacher, M., Hubner, R., \& Schlosser, J. (2011). Monetary incentives in speeded perceptual decision: effects of penalizing errors versus slow responses. Front Psychol, 2, 248.

Dimigen, O., Sommer, W., Hohlfeld, A., Jacobs, A. M., \& Kliegl, R. (2011). Coregistration of eye movements and EEG in natural reading: analyses and review. Journal of Experimental Psychology: General, 140, 552.

Eckstein, M. K., Guerra-Carrillo, B., Singley, A. T. M., \& Bunge, S. A. (2017). Beyond eye gaze: What else can eyetracking reveal about cognition and cognitive development? Developmental cognitive neuroscience, 25, 69-91.

Gilzenrat, M. S., Nieuwenhuis, S., Jepma, M., \& Cohen, J. D. (2010). Pupil diameter tracks changes in control state predicted by the adaptive gain theory of locus coeruleus function. Cogn Affect Behav Neurosci, 10, 252-269.

Goldstein, R. Z., Cottone, L. A., Jia, Z., Maloney, T., Volkow, N. D., \& Squires, N. K. (2006). The effect of graded monetary reward on cognitive event-related potentials and behavior in young healthy adults. International Journal of Psychophysiology, 62, 272-279.

Grent-'t-Jong, T., \& Woldorff, M. G. (2007). Timing and Sequence of Brain Activity in Top-Down Control of Visual-Spatial Attention. PLoS Biol, 5, e12.

Hong, L., Walz, J. M., \& Sajda, P. (2014). Your eyes give you away: prestimulus changes in pupil diameter correlate with poststimulus task-related EEG dynamics. PLoS One, 9, e91321. 
Hopf, J. M., Vogel, E., Woodman, G., Heinze, H. J., \& Luck, S. J. (2002). Localizing Visual Discrimination Processes in Time and Space. J Neurophysiol, 88, 20882095.

Hubner, R., \& Schlosser, J. (2010). Monetary reward increases attentional effort in the flanker task. Psychon Bull Rev, 17, 821-826.

Hughes, G., Mathan, S., \& Yeung, N. (2013). EEG indices of reward motivation and target detectability in a rapid visual detection task. Neurolmage, 64, 590-600.

Inzlicht, M., Shenhav, A., \& Olivola, C. Y. (in press). The Effort Paradox: Effort Is Both Costly and Valued. Trends Cogn Sci.

Janssens, C., De Loof, E., Pourtois, G., \& Verguts, T. (2016). The time course of cognitive control implementation. Psychonomic Bulletin \& Review, 23, 12661272.

Jenkins Jr, G. D., Mitra, A., Gupta, N., \& Shaw, J. D. (1998). Are financial incentives related to performance? A meta-analytic review of empirical research. Journal of applied psychology, 83, 777.

Jimura, K., Locke, H. S., \& Braver, T. S. (2010). Prefrontal cortex mediation of cognitive enhancement in rewarding motivational contexts. PNAS, 107, 8871-8876.

Kahneman, D., \& Beatty, J. (1966). Pupil diameter and load on memory. Science, 154, 1583-1585.

Kool, W., McGuire, J. T., Rosen, Z. B., \& Botvinick, M. M. (2010). Decision making and the avoidance of cognitive demand. J Exp Psychol Gen, 139, 665-682.

Kouneiher, F., Charron, S., \& Koechlin, E. (2009). Motivation and cognitive control in the human prefrontal cortex. Nat Neurosci, 12, 939-945.

Krebs, R. M., Boehler, C. N., Appelbaum, L. G., \& Woldorff, M. G. (2013). Reward associations reduce behavioral interference by changing the temporal dynamics of conflict processing. PLoS One, 8, e53894.

Krebs, R. M., Boehler, C. N., Egner, T., \& Woldorff, M. G. (2011). The neural underpinnings of how reward associations can both guide and misguide attention. J Neurosci, 31, 9752-9759.

Krebs, R. M., Boehler, C. N., \& Woldorff, M. G. (2010). The influence of reward associations on conflict processing in the Stroop task. Cognition, 117, 341-347.

Krebs, R. M., Hopf, J.-M., \& Boehler, C. N. (2016). Within-trial effects of stimulus-reward associations. In T. Braver (Ed.), Motivation and cognitive control (pp. 65-82): Psychology Press.

Krebs, R. M., \& Woldorff, M. G. (2017). Cognitive Control and Reward. The Wiley Handbook of Cognitive Control, 422-439.

Laeng, B., Orbo, M., Holmlund, T., \& Miozzo, M. (2011). Pupillary Stroop effects. Cogn Process, 12, 13-21.

Langford, Z. D., Schevernels, H., \& Boehler, C. N. (2016). Motivational context for response inhibition influences proactive involvement of attention. Sci Rep, 6 , 35122.

Linssen, A. M., Vuurman, E. F., Sambeth, A., Nave, S., Spooren, W., Vargas, G., Santarelli, L., \& Riedel, W. J. (2011). Contingent negative variation as a dopaminergic biomarker: evidence from dose-related effects of methylphenidate. Psychopharmacology (Berl), 218, 533-542. 
Locke, H. S., \& Braver, T. S. (2008). Motivational influences on cognitive control: behavior, brain activation, and individual differences. Cogn Affect Behav Neurosci, 8, 99-112.

Massar, S. A., Lim, J., Sasmita, K., \& Chee, M. W. (2016). Rewards boost sustained attention through higher effort: A value-based decision making approach. Biol Psychol, 120, 21-27.

Morey, R. D. (2008). Confidence intervals from normalized data: A correction to Cousineau (2005). reason, 4, 61-64.

Murphy, P. R., O'connell, R. G., O'sullivan, M., Robertson, I. H., \& Balsters, J. H. (2014). Pupil diameter covaries with BOLD activity in human locus coeruleus. Hum Brain Mapp, 35, 4140-4154.

Murphy, P. R., Robertson, I. H., Balsters, J. H., \& O'Connell R, G. (2011). Pupillometry and P3 index the locus coeruleus-noradrenergic arousal function in humans. Psychophysiology, 48, 1532-1543.

Nieuwenhuis, S., Forstmann, B. U., \& Wagenmakers, E. J. (2011). Erroneous analyses of interactions in neuroscience: a problem of significance. Nat Neurosci, 14, 1105-1107.

Notebaert, W., \& Braem, S. (2015). Parsing the effects of reward on cognitive control. Motivation and cognitive control, 105-122.

Padmala, S., \& Pessoa, L. (2011). Reward reduces conflict by enhancing attentional control and biasing visual cortical processing. J Cogn Neurosci, 23, 3419-3432.

Paschke, L. M., Walter, H., Steimke, R., Ludwig, V. U., Gaschler, R., Schubert, T., \& Stelzel, C. (2015). Motivation by potential gains and losses affects control processes via different mechanisms in the attentional network. Neurolmage, 111, 549-561.

Pernet, C. R., Chauveau, N., Gaspar, C., \& Rousselet, G. A. (2011). LIMO EEG: a toolbox for hierarchical LInear MOdeling of ElectroEncephaloGraphic data. Comput Intell Neurosci, 2011, 831409.

Polich, J. (2007). Updating P300: an integrative theory of P3a and P3b. Clin Neurophysiol, 118, 2128-2148.

Reimer, J., McGinley, M. J., Liu, Y., Rodenkirch, C., Wang, Q., McCormick, D. A., \& Tolias, A. S. (2016). Pupil fluctuations track rapid changes in adrenergic and cholinergic activity in cortex. Nat Commun, 7, 13289.

Richter, M., \& Slade, K. (2017). Interpretation of physiological indicators of motivation: Caveats and recommendations. Int J Psychophysiol, 119, 4-10.

Schevernels, H., Bombeke, K., Van der Borght, L., Hopf, J. M., Krebs, R. M., \& Boehler, C. N. (2015). Electrophysiological evidence for the involvement of proactive and reactive control in a rewarded stop-signal task. Neurolmage, 121, 115-125.

Schevernels, H., Krebs, R. M., Santens, P., Woldorff, M. G., \& Boehler, C. N. (2014). Task preparation processes related to reward prediction precede those related to task-difficulty expectation. Neurolmage, 84, 639-647.

Seamans, J. K., \& Yang, C. R. (2004). The principal features and mechanisms of dopamine modulation in the prefrontal cortex. Prog Neurobiol, 74, 1-58.

Shenhav, A., Musslick, S., Lieder, F., Kool, W., Griffiths, T. L., Cohen, J. D., \& Botvinick, M. M. (2017). Toward a Rational and Mechanistic Account of Mental Effort. Annual Review of Neurosciences, 40, 99-124. 
Shine, J. M., Bissett, P. G., Bell, P. T., Koyejo, O., Balsters, J. H., Gorgolewski, K. J., Moodie, C. A., \& Poldrack, R. A. (2016). The Dynamics of Functional Brain Networks: Integrated Network States during Cognitive Task Performance. Neuron, 92, 544-554.

Tecce, J. J. (1972). Contingent negative variation (CNV) and psychological processes in man. Psychological Bulletin, 77, 73.

van den Berg, B., Krebs, R. M., Lorist, M. M., \& Woldorff, M. G. (2014). Utilization of reward-prospect enhances preparatory attention and reduces stimulus conflict. Cognitive, Affective, \& Behavioral Neuroscience, 14, 561-577.

van Dijk, H., Schoffelen, J. M., Oostenveld, R., \& Jensen, O. (2008). Prestimulus oscillatory activity in the alpha band predicts visual discrimination ability. $J$ Neurosci, 28, 1816-1823.

Varazzani, C., San-Galli, A., Gilardeau, S., \& Bouret, S. (2015). Noradrenaline and dopamine neurons in the reward/effort trade-off: a direct electrophysiological comparison in behaving monkeys. J Neurosci, 35, 7866-7877.

Verguts, T., Vassena, E., \& Silvetti, M. (2015). Adaptive effort investment in cognitive and physical tasks: a neurocomputational model. Front Behav Neurosci, 9, 57.

Vogel, E. K., \& Luck, S. (2000). The visual N1 component as an index of a discrimination process. Psychophysiology, 37, 190-203.

van der Wel, P., \& van Steenbergen, H. (2018). Pupil dilation as an index of effort in cognitive control tasks: A review. Psychonomic Bulletin \& Review.

Westbrook, A., \& Braver, T. S. (2015). Cognitive effort: A neuroeconomic approach. Cogn Affect Behav Neurosci, 15, 395-415.

Westbrook, A., \& Braver, T. S. (2016). Dopamine Does Double Duty in Motivating Cognitive Effort. Neuron, 89, 695-710.

Wolff, N., Muckschel, M., Ziemssen, T., \& Beste, C. (2018). The role of phasic norepinephrine modulations during task switching: evidence for specific effects in parietal areas. Brain Struct Funct, 223, 925-940.

Xing, B., Li, Y. C., \& Gao, W. J. (2016). Norepinephrine versus dopamine and their interaction in modulating synaptic function in the prefrontal cortex. Brain Res, 1641, 217-233. 